\title{
A Simulation-Based Study for Progressive Estimation of Population Mean through Traditional and Nontraditional Measures in Stratified Random Sampling
}

\author{
Maria Javed $\left(\mathbb{D},{ }^{1}\right.$ Muhammad Irfan $\left(\mathbb{D},{ }^{1}\right.$ Sajjad Haider Bhatti $\mathbb{D}^{\mathrm{D}},{ }^{1}$ and Ronald Onyango $\mathbb{D}^{2}$ \\ ${ }^{1}$ Department of Statistics, Government College University, Faisalabad, Pakistan \\ ${ }^{2}$ Department of Applied Statistics, Financial Mathematics and Actuarial Science, \\ Jaramogi Oginga Odinga University of Science and Technology, Bondo, Kenya
}

Correspondence should be addressed to Ronald Onyango; assangaronald@gmail.com

Received 3 October 2021; Accepted 27 November 2021; Published 20 December 2021

Academic Editor: Niansheng Tang

Copyright (C) 2021 Maria Javed et al. This is an open access article distributed under the Creative Commons Attribution License, which permits unrestricted use, distribution, and reproduction in any medium, provided the original work is properly cited.

This study suggests a new optimal family of exponential-type estimators for estimating population mean in stratified random sampling. These estimators are based on the traditional and nontraditional measures of auxiliary information. Expressions for the bias, mean square error, and minimum mean square error of the proposed estimators are derived up to first order of approximation. It is observed that proposed estimators perform better than the traditional estimators (unbiased, combined ratio, and combined regression) and other recent estimators. A real dataset is used to highlight the applicability of proposed estimators. In addition, a simulation study is carried out to assess the performance of new family as compared to other estimators.

\section{Introduction}

Nowadays, it is common practice to use the auxiliary/ancillary information to boost the efficiency of estimators in survey sampling. Most of the researchers only deal with the traditional information of auxiliary variable(s) such as standard deviation, coefficient of variation, coefficient of skewness, coefficient of kurtosis, and coefficient of correlation. Having edge of this traditional information, many authors have been trying to explore new optimal estimators and families of estimators for estimating population mean under stratified random sampling. Stratified random sampling has often proved needful in improving the precision of estimators over simple random sampling, for instance, see works of Kadilar and Cingi [1,2], Koyuncu and Kadilar [3, 4], Singh and Vishwakarma [5-7], Shabbir and Gupta [8], Haq and Shabbir [9], Singh and Solanki [10], Yadav et al. [11], Solanki and Singh [10, 12], Javed et al. [13], and Javed and Irfan [14].

The motivation behind this article is to utilize the nontraditional information as well as the traditional information of the auxiliary variable to progress the estimation of population mean in stratified random sampling. This idea is initiated first time in this article under stratified random sampling.

Nontraditional information includes quartile deviation, midrange, interquartile range, quartile average, decile mean, tri-mean, Hodges-Lehmann estimator, and L-moments of an auxiliary variable. L-moments are determined by linear combinations of the expected values of the order statistics (for detail, check the works of Hosking [15] and Shahzad et al. [16]). Furthermore, efficiency of the estimators is uncertain in the occurrence of the extreme values in the dataset. Some of the above nontraditional measures such as decile mean, Hodges-Lehmann estimator, and tri-mean are robust measures. Utilizing these measures, we can well cope with the extreme values/outliers in the dataset. In addition, L-moments also are used to reduce the negative effect of outliers on the estimators.

Rest of the article is organized in the following way. Section 2 presents the useful notations. Section 3 gives comprehensive detail of existing families of estimators. Section 4 suggests a new optimal family of estimators for estimating population mean using traditional and nontraditional measures of auxiliary variable. Expressions for the 
bias, mean squared error (MSE) and minimum MSE of this family are derived up to first degree of approximation in the same section. A real dataset is used in Section 5 to check the potential of new estimators as compared to existing ones. In Section 6, the performance of suggested family is evaluated by carrying out a simulation study using the same dataset used in Section 5. Section 7 contains the final discussion.

\section{Useful Notations}

Let us consider a finite population $U=\left\{U_{1}, U_{2}, U_{3}, \ldots, U_{N}\right\}$ of size $N$, and it can be stratified into $L$ homogenous strata with $h^{\text {th }}$ stratum containing $N_{h},(h=1,2, \ldots, L)$ units subject to the restriction that $\sum_{h=1}^{L} N_{h}=N$. A sample of size $n_{h}$ is drawn under simple random sampling without replacement (SRSWOR) from $h^{\text {th }}$ stratum such that $\sum_{h=1}^{L} n_{h}=n$. Consider the $N_{h}$ pairs of observations $\left(y_{h i}, x_{h i}\right), i=1,2,3, \ldots, N_{h}$, made from $h^{\text {th }}$ stratum for the study and auxiliary variables, respectively.

Furthermore, let $\bar{Y}=\bar{Y}_{s t}=\sum_{h=1}^{L} W_{h} \bar{Y}_{h}$ and $\bar{y}_{s t}=\sum_{h=1}^{L}$ $W_{h} \bar{y}_{h}$ be the population and sample means of $y$, respectively, where $\bar{Y}_{h}=\sum_{i=1}^{N_{h}}\left(y_{h i} / N_{h}\right), \bar{y}_{h}=\sum_{i=1}^{n_{h}}\left(y_{h i} / n_{h}\right)$, and $W_{h}=$ $\left(N_{h} / N\right)$ are the population mean, sample mean, and the weight of $h^{\text {th }}$ stratum, respectively. Following the same lines, $\bar{X}_{s t}, \bar{x}_{s t}, \bar{X}_{h}$ and $\bar{x}_{h}$ can be defined for the auxiliary variable $x$.

To derive the expressions for the bias, mean square error (MSE), and minimum mean square error of the existing and proposed estimators, we consider the following relative error terms along with their expectations as

$$
\begin{gathered}
\zeta_{0}=\frac{\bar{y}_{s t}-\bar{Y}}{\bar{Y}}, \\
\zeta_{1}=\frac{\bar{x}_{s t}-\bar{X}}{\bar{X}},
\end{gathered}
$$

such that

$$
\begin{aligned}
E\left(\zeta_{i}\right) & =0 \text { for } i=0,1, \\
V_{m n} & =\sum_{h=1}^{L} W_{h}^{m+n} \frac{E\left[\left(\bar{y}_{h}-\bar{Y}_{h}\right)^{m}\left(\bar{x}_{h}-\bar{X}_{h}\right)^{n}\right]}{\bar{Y}^{m} \bar{X}^{n}} .
\end{aligned}
$$

From (2), we can write as below:

$$
\begin{gathered}
E\left(\zeta_{0}^{2}\right)=\frac{\sum_{h=1}^{L} W_{h}^{2} \phi_{h} S_{y h}^{2}}{\bar{Y}^{2}}=V_{20}, \\
E\left(\zeta_{1}^{2}\right)=\frac{\sum_{h=1}^{L} W_{h}^{2} \phi_{h} S_{x h}^{2}}{\bar{X}^{2}}=V_{02}, \\
E\left(\zeta_{0} \zeta_{1}\right)=\frac{\sum_{h=1}^{L} W_{h}^{2} \phi_{h} S_{y x h}}{\overline{Y X}}=V_{11},
\end{gathered}
$$

where

$$
\begin{aligned}
\phi_{h} & =\frac{\left(1-f_{h}\right)}{n_{h}}, \\
f_{h} & =\frac{n_{h}}{N_{h}}, \\
S_{y h}^{2} & =\frac{\sum_{i=1}^{N_{h}}\left(y_{h i}-\bar{Y}_{h}\right)^{2}}{N_{h}-1}, \\
S_{x h}^{2} & =\sum_{i=1}^{N_{h}} \frac{\left(x_{h i}-\bar{X}_{h}\right)^{2}}{N_{h}-1}, \\
S_{y x h} & =\frac{\sum_{i=1}^{N_{h}}\left(y_{h i}-\bar{Y}_{h}\right)\left(x_{h i}-\bar{X}_{h}\right)}{N_{h}-1} .
\end{aligned}
$$

Some other formulas for $h^{\text {th }}$ stratum, under stratified random sampling are listed below:

$$
\begin{aligned}
& \text { Coefficient of Varaiation } C_{x h}=\frac{S_{x h}}{\bar{X}_{h}} \text {, } \\
& \text { Coefficient of Skewness } \beta_{1 x h}=\frac{N \sum_{i=1}^{N}\left(x_{i h}-\bar{X}_{h}\right)^{3}}{(N-1)(N-2) S_{x h}^{3}}, \\
& \text { Coefficient of kurtosis } \beta_{2 x h}=\frac{N(N+1) \sum_{i=1}^{N}\left(x_{i h}-\bar{X}_{h}\right)^{4}}{(N-1)(N-2)(N-3) S_{x h}^{4}}-\frac{3(N-1)^{2}}{(N-2)(N-3)} \text {, } \\
& \text { Quartile deviation } Q D_{x h}=\frac{Q_{3 h}-Q_{1 h}}{2} \text {, } \\
& \text { Midrange } \mathrm{MR}_{x h}=\frac{x_{(1) h}+x_{(N) h}}{2}, \\
& \text { Interquartile range } Q R_{x h}=Q_{3 h}-Q_{1 h} \text {, } \\
& \text { Quartile average } Q A_{x h}=\frac{Q_{3 h}+Q_{1 h}}{2} \text {, } \\
& \text { Tri-mean } \mathrm{TM}_{x h}=\frac{Q_{1 h}+2 Q_{2 h}+Q_{3 h}}{4}, \\
& \text { Hodge - Lehmann } \mathrm{HL}_{x h}=\operatorname{Median}\left(\frac{x_{j h}+x_{k h}}{2}\right), \quad 1 \leq j h \leq k h \leq N \text {, }
\end{aligned}
$$


where $Q_{1}, Q_{2}$, and $Q_{3}$ are the first, second, and third quartiles, respectively, $x_{(1)}$ is the minimum value, and $x_{(N)}$ is the maximum value of the data.

\section{Some Existing Estimators/Classes of Estimators}

This section gives a brief introduction of some well-known estimators/classes of estimators from the literature.

3.1. Usual Estimators. In stratified random sampling usual unbiased $\overline{\bar{Y}}_{s t}$, combined ratio $\bar{Y}_{C R}$ and combined regression $\overline{\bar{Y}}_{\text {CReg }}$ estimators and their MSEs are detailed below:

$$
\begin{aligned}
\hat{\bar{Y}}_{s t} & =\sum_{h=1}^{L} W_{h} \bar{y}_{h}, \\
\hat{\bar{Y}}_{C R} & =\bar{y}_{s t}\left(\frac{\bar{X}}{\bar{x}_{s t}}\right), \\
\hat{\bar{Y}}_{C \text { Reg }} & =\left[\bar{y}_{s t}+\widehat{b}\left(\bar{X}-\bar{x}_{s t}\right)\right], \text { with } \widehat{b}=\frac{\sum_{h=1}^{L} W_{h}^{2} \phi_{h} S_{y x h}}{\sum_{h=1}^{L} W_{h}^{2} \phi_{h} S_{x h}^{2}}, \\
\operatorname{MSE}\left(\hat{\bar{Y}}_{s t}\right) & =\operatorname{Var}\left(\hat{\bar{Y}}_{s t}\right)=\bar{Y}^{2} V_{20}, \\
\operatorname{MSE}\left(\hat{\bar{Y}}_{C R}\right) & =\bar{Y}^{2}\left(V_{20}+V_{02}-2 V_{11}\right), \\
\operatorname{MSE}\left(\hat{\bar{Y}}_{C R e g}\right) & =\bar{Y}^{2} V_{20}\left(1-\rho_{s t}^{2}\right),
\end{aligned}
$$

where $\rho_{s t}=\sum_{h=1}^{L} W_{h} \rho_{y x h}=\sum_{h=1}^{L} W_{h}^{2} \phi_{h} S_{y x h} / \sqrt{\sum_{h=1}^{L} W_{h}^{2} \phi_{h} S_{y h}^{2}}$ $\sqrt{\sum_{h=1}^{L} W_{h}^{2} \phi_{h} S_{x h}^{2}}=V_{11} / \sqrt{V_{20}} \sqrt{V_{02}}$.

Bahl and Tuteja [17] suggested ratio and product exponential-type estimators for population mean under stratified random sampling as

$$
\begin{aligned}
& \hat{\bar{Y}}_{B T, R e}=\bar{y}_{s t} \exp \left(\frac{\bar{X}-\bar{x}_{s t}}{\bar{X}+\bar{x}_{s t}}\right), \\
& \hat{\bar{Y}}_{B T, P e}=\bar{y}_{s t} \exp \left(\frac{\bar{x}_{s t}-\bar{X}}{\bar{x}_{s t}+\bar{X}}\right) .
\end{aligned}
$$

Average of (7) and (8) can be written as

$$
\begin{aligned}
& \hat{\bar{Y}}_{B T, \mathrm{Avg}}=\frac{\bar{y}_{s t}}{2}\left\{\exp \left(\frac{\bar{X}-\bar{x}_{s t}}{\bar{X}+\bar{x}_{s t}}\right)+\exp \left(\frac{\bar{x}_{s t}-\bar{X}}{\bar{x}_{s t}+\bar{X}}\right)\right\}, \\
& \operatorname{MSE}\left(\hat{\bar{Y}}_{B T, R e}\right) \cong \bar{Y}^{2}\left(V_{20}+\frac{V_{02}}{4}-V_{11}\right), \\
& \operatorname{MSE}\left(\hat{\bar{Y}}_{B T, P e}\right) \cong \bar{Y}^{2}\left(V_{20}+\frac{V_{02}}{4}+V_{11}\right) .
\end{aligned}
$$

3.2. Koyuncu and Kadilar [3]. To estimate the population mean under stratified random sampling, a family of ratio estimators was introduced by Koyuncu and Kadilar [3] as below:

$$
\hat{\bar{Y}}_{K}=\bar{y}_{s t}\left[\frac{a_{s t} \bar{X}+b_{s t}}{\gamma\left(a_{s t} \bar{x}_{s t}+b_{s t}\right)+(1-\gamma)\left(a_{s t} \bar{X}+b_{s t}\right)}\right]^{g},
$$

where $\gamma$ and $g$ are suitable constants and $a_{s t}(\neq 0)$ and $b_{s t}$ are either real numbers or functions of known parameters of the auxiliary variable such as coefficient of skewness, coefficient of kurtosis, coefficient of variation, and coefficient of correlation.

Up to the first order of approximation, the bias and MSE of $\overline{\bar{Y}}_{K}$ are given by

$$
\begin{aligned}
& \operatorname{Bias}\left(\hat{\bar{Y}}_{K}\right) \cong \bar{Y}\left(\frac{g(g+1)}{2} \gamma^{2} \eta^{2} V_{02}-g \gamma \eta V_{11}\right), \\
& \operatorname{MSE}\left(\hat{\bar{Y}}_{K}\right) \cong \bar{Y}^{2}\left(V_{20}+g^{2} \gamma^{2} \eta^{2} V_{02}-2 g \gamma \eta V_{11}\right), \\
& \text { where } \eta=\frac{a_{s t} \bar{X}}{a_{s t} \bar{X}+b_{s t}} \\
& \operatorname{At}(g \gamma \eta)_{\text {opt }}= \frac{V_{11}}{V_{02}}, \text { the minimum MSE } \hat{\bar{Y}}_{\mathrm{K}} \text { is given as, } \\
& \operatorname{MSE}_{\text {min }}\left(\hat{\bar{Y}}_{K}\right) \cong \bar{Y}^{2} V_{20}\left(1-\rho_{s t}^{2}\right) .
\end{aligned}
$$

3.3. Koyuncu and Kadilar [4]. Koyuncu and Kadilar [4] considered the ratio estimator of Gupta and Shabbir [18] and suggested an improved estimator defined as below:

$$
\widehat{\bar{Y}}_{K K}=\left[\theta_{1} \bar{y}_{s t}+\theta_{2}\left(\bar{X}-\bar{x}_{s t}\right)\right]\left(\frac{a_{s t} \bar{X}+b_{s t}}{a_{s t} \bar{x}_{s t}+b_{s t}}\right),
$$

where $\theta_{1}$ and $\theta_{2}$ are suitably chosen weights.

Given below are the expressions, up to first degree of approximation, for the bias and MSE of $\hat{\bar{Y}}_{K K}$, respectively:

$$
\operatorname{Bias}\left(\widehat{\bar{Y}}_{K K}\right) \cong-\bar{Y}+\bar{X} \eta \theta_{2} V_{02}+\bar{Y} \theta_{1}\left(1+\eta^{2} V_{02}-\eta V_{11}\right),
$$

$$
\begin{aligned}
\operatorname{MSE}\left(\hat{\bar{Y}}_{K K}\right) \cong & \bar{Y}^{2}+\bar{X} \theta_{2}\left(-2 \bar{Y} \eta+\bar{X} \theta_{2}\right) V_{02}-2 \bar{Y} \theta_{1} \\
& \cdot\left\{\bar{Y}+\eta\left(\bar{Y} \eta-2 \bar{X} \theta_{2}\right) V_{02}+\left(-\bar{Y} \eta+\bar{X} \theta_{2}\right) V_{11}\right\} \\
& +\bar{Y}^{2} \theta_{1}^{2}\left(1+3 \eta^{2} V_{02}-4 \eta V_{11}+V_{20}\right) .
\end{aligned}
$$

The suitable weights of $\theta_{1}$ and $\theta_{2}$ are given by 


$$
\begin{aligned}
& \theta_{1}=\frac{\left(-1+\eta^{2} V_{02}\right) V_{02}}{\eta^{2} V_{02}^{2}+V_{11}^{2}-V_{02}\left(1+V_{20}\right)}, \\
& \theta_{2}=-\frac{\bar{Y}\left\{V_{11}+\eta\left(\eta^{2} V_{02}^{2}-V_{11}^{2}+V_{02}\left(-1-\eta V_{11}+V_{20}\right)\right)\right\}}{\bar{X}\left\{\eta^{2} V_{02}^{2}+V_{11}^{2}-V_{02}\left(1+V_{20}\right)\right\}} .
\end{aligned}
$$

Inserting the above weights of $\theta_{1}$ and $\theta_{2}$ in (14), we get the minimum MSE of $\bar{Y}_{K K}$ as

$$
\operatorname{MSE}_{\min }\left(\hat{\bar{Y}}_{K K}\right) \cong \frac{\bar{Y}^{2}\left(-1+\eta^{2} V_{02}\right)\left(-V_{11}^{2}+V_{02} V_{20}\right)}{\eta^{2} V_{02}^{2}+V_{11}^{2}-V_{02}\left(1+V_{20}\right)}
$$

3.4. Shabbir and Gupta [8]. Given below is a ratio-type estimator suggested by Shabbir and Gupta [8] in stratified random sampling:

$$
\widehat{\bar{Y}}_{S G}=\left[\theta_{3} \bar{y}_{s t}+\theta_{4}\left(\bar{X}-\bar{x}_{s t}\right)\right] \exp \left(\frac{\bar{A}-\bar{a}_{s t}}{\bar{A}+\bar{a}_{s t}}\right)
$$

where $\theta_{3}$ and $\theta_{4}$ are the constants to be determined. Also, we consider that

$$
\begin{gathered}
a_{h i}=x_{h i}+N \bar{X}, \\
\bar{a}_{s t}=\bar{x}_{s t}+N \bar{X}, \\
\bar{A}=(1+N) \bar{X} .
\end{gathered}
$$

Expressions for the bias and the MSE of $\widehat{\bar{Y}}_{S G}$, respectively, are given below:

$$
\begin{aligned}
\operatorname{Bias}\left(\hat{\bar{Y}}_{S G}\right) \cong & \frac{4(1+N)\left\{-2(1+N) \bar{Y}+\bar{X} \theta_{4} V_{02}\right\}+\bar{Y} \theta_{3}\left\{3 V_{02}+4(1+N)\left(2+2 N-V_{11}\right)\right\}}{8(1+N)^{2}}, \\
\operatorname{MSE}\left(\hat{\bar{Y}}_{S G}\right) \cong & \bar{Y}^{2}\left(\theta_{3}-1\right)^{2}+\frac{\bar{Y}^{2} V_{11} \theta_{3}}{(1+N)}-\frac{3 \bar{Y}^{2} V_{02} \theta_{3}}{4(1+N)^{2}}+\bar{Y}^{2} V_{02} \theta_{3}^{2}-\frac{2 \bar{Y}^{2} V_{11} \theta_{3}^{2}}{(1+N)}+\frac{\bar{Y}^{2} V_{02} \theta_{3}^{2}}{(1+N)^{2}} \\
& -\frac{\overline{X Y} V_{02} \theta_{4}}{(1+N)}-2 \overline{X Y} V_{11} \theta_{3} \theta_{4}+\frac{2 \overline{X Y} V_{02} \theta_{3} \theta_{4}}{(1+N)}+\bar{X}^{2} V_{02} \theta_{4}^{2} .
\end{aligned}
$$

The suitable weights of $\theta_{3}$ and $\theta_{4}$ are given as

$$
\begin{aligned}
& \theta_{3}=\frac{V_{02}\left\{8(1+N)^{2}-V_{02}\right\}}{8(1+N)^{2}\left\{-V_{11}^{2}+V_{02}\left(1+V_{20}\right)\right\}}, \\
& \theta_{4}=\frac{\bar{Y}\left[V_{02}^{2}+4(1+N)^{2}\left(2+2 N-V_{11}\right) V_{11}+(1+N) V_{02}\left\{-V_{11}^{2}+4(1+N)\left(V_{20}-1\right)\right\}\right]}{8 \bar{X}(1+N)^{3}\left\{-V_{11}^{2}+V_{02}\left(1+V_{20}\right)\right\}} .
\end{aligned}
$$

Putting weights of $\theta_{3}$ and $\theta_{4}$ in (20), we have minimum MSE of $\bar{Y}_{S G}$ as

$$
\operatorname{MSE}_{\min }\left(\widehat{\bar{Y}}_{S G}\right) \cong \frac{\bar{Y}^{2}\left[V_{02}^{3}+64(1+N)^{4} V_{11}^{2}+16(1+N)^{2} V_{02}^{2} V_{20}-16(1+N)^{2} V_{02}\left\{V_{11}^{2}+4(1+N)^{2} V_{20}\right\}\right]}{64(1+N)^{4}\left\{V_{11}^{2}-V_{02}\left(1+V_{20}\right)\right\}}
$$


3.5. Haq and Shabbir [9]. Haq and Shabbir [9] proposed two exponential ratio-type families of estimators detailed below:

$$
\begin{aligned}
\hat{\bar{Y}}_{H S 1}= & {\left[\theta_{5} \bar{y}_{s t}+\theta_{6}\left(\bar{X}-\bar{x}_{s t}\right)\right] \exp \left(\frac{a_{s t} \bar{X}+b_{s t}}{\gamma\left(a_{s t} \bar{x}_{s t}+b_{s t}\right)+(1-\gamma)\left(a_{s t} \bar{X}+b_{s t}\right)}-1\right), } \\
\hat{\bar{Y}}_{H S 2}= & {\left[\theta_{7} \bar{y}_{s t}+\theta_{8}\left(\bar{X}-\bar{x}_{s t}\right)\right] \exp \left(\frac{a_{s t} \bar{X}+b_{s t}}{\gamma\left(a_{s t} \bar{x}_{s t}+b_{s t}\right)+(1-\gamma)\left(a_{s t} \bar{X}+b_{s t}\right)}-1\right) } \\
& \times\left\{\left(\frac{1}{2}\right)\left(\frac{a_{s t} \bar{X}+b_{s t}}{\gamma\left(a_{s t} \bar{x}_{s t}+b_{s t}\right)+(1-\gamma)\left(a_{s t} \bar{X}+b_{s t}\right)}+\frac{\gamma\left(a_{s t} \bar{x}_{s t}+b_{s t}\right)+(1-\gamma)\left(a_{s t} \bar{X}+b_{s t}\right)}{a_{s t} \bar{X}+b_{s t}}\right)\right\}^{2},
\end{aligned}
$$

where $\theta_{5}, \theta_{6}, \theta_{7}$, and $\theta_{8}$ are the suitable constants.

$\widehat{\bar{Y}}$ Given below are the expressions for bias and MSE of $\widehat{\bar{Y}}_{H S 1}$ and $\widehat{\bar{Y}}_{H S 2}$, respectively:

$$
\begin{aligned}
\operatorname{Bias}\left(\hat{\bar{Y}}_{H S 1}\right) \cong & -\bar{Y}+\bar{X} \psi \theta_{6} V_{02}+\theta_{5}\left(\bar{Y}+\frac{3 \bar{Y} \psi^{2} V_{02}}{2}-\bar{Y} \psi V_{11}\right) \\
\operatorname{Bias}\left(\hat{\bar{Y}}_{H S 2}\right) \cong & -\bar{Y}+\bar{X} \psi \theta_{8} V_{02}+\theta_{7}\left(\bar{Y}+\frac{5 \bar{Y} \psi^{2} V_{02}}{2}-\bar{Y} \psi V_{11}\right) \\
\operatorname{MSE}\left(\widehat{\bar{Y}}_{H S 1}\right) \cong & \bar{Y}^{2}+\bar{X} \theta_{6}\left(-2 \bar{Y} \psi+\bar{X} \theta_{6}\right) V_{02}+\bar{Y} \theta_{5}\left\{-2 \bar{Y}+\psi\left(-3 \bar{Y} \psi+4 \bar{X} \theta_{6}\right) V_{02}+2\left(\bar{Y} \psi-\bar{X} \theta_{6}\right) V_{11}\right\} \\
& +\bar{Y}^{2} \theta_{5}^{2}\left(1+4 \psi^{2} V_{02}-4 \psi V_{11}+V_{20}\right), \\
\operatorname{MSE}\left(\hat{\bar{Y}}_{H S 2}\right) \cong & \bar{Y}^{2}+\bar{X} \theta_{8}\left(-2 \bar{Y} \psi+\bar{X} \theta_{8}\right) V_{02}+\bar{Y} \theta_{7}\left\{-2 \bar{Y}+\psi\left(-5 \bar{Y} \psi+4 \bar{X} \theta_{8}\right) V_{02}+2\left(\bar{Y} \psi-\bar{X} \theta_{8}\right) V_{11}\right\} \\
& +\bar{Y}^{2} \theta_{7}^{2}\left(1+6 \psi^{2} V_{02}-4 \psi V_{11}+V_{20}\right),
\end{aligned}
$$

where $\psi=\gamma \eta$ and $\eta$ is defined earlier.

The weights of $\theta_{5}, \theta_{6}, \theta_{7}$, and $\theta_{8}$ are determined as below:

$$
\begin{aligned}
& \theta_{5}=\frac{V_{02}\left(2-\psi^{2} V_{02}\right)}{2\left\{-V_{11}^{2}+V_{02}\left(1+V_{20}\right)\right\}}, \\
& \theta_{6}=\frac{\bar{Y}\left\{2 \psi^{3} V_{02}^{2}-2 V_{11}\left(-1+\psi V_{11}\right)-\psi V_{02}\left(2+\psi V_{11}-2 V_{20}\right)\right\}}{2 \bar{X}\left\{-V_{11}^{2}+V_{02}\left(1+V_{20}\right)\right\}}, \\
& \theta_{7}=\frac{V_{02}\left(2+\psi^{2} V_{02}\right)}{2\left\{-V_{11}^{2}+V_{02}\left(1+2 \psi^{2} V_{02}+V_{20}\right)\right\}}, \\
& \theta_{8}=\frac{\bar{Y}\left\{2 \psi^{3} V_{02}^{2}-2 V_{11}\left(-1+\psi V_{11}\right)+\psi V_{02}\left(-2+\psi V_{11}+2 V_{20}\right)\right\}}{2 \bar{X}\left\{-V_{11}^{2}+V_{02}\left(1+2 \psi^{2} V_{02}+V_{20}\right)\right\}} .
\end{aligned}
$$


By substituting values of $\theta_{5}$ and $\theta_{6}$ in (25) and $\theta_{7}$ and $\theta_{8}$ in (26), we get minimum MSE of $\widehat{Y}_{H S 1}$ and $\bar{Y}_{H S 2}$, respectively:

$$
\begin{aligned}
& \operatorname{MSE}_{\text {min }}\left(\widehat{\bar{Y}}_{H S 1}\right) \cong \frac{\bar{Y}^{2}\left[4 V_{11}^{2}+V_{02}\left\{\psi^{4} V_{02}^{2}-4 \psi^{2} V_{11}^{2}+4\left(-1+\psi^{2} V_{02}\right) V_{20}\right\}\right]}{4\left\{V_{11}^{2}-V_{02}\left(1+V_{20}\right)\right\}}, \\
& \operatorname{MSE}_{\text {min }}\left(\hat{\bar{Y}}_{H S 2}\right) \cong \frac{\bar{Y}^{2}\left[4 V_{11}^{2}+V_{02}\left\{9 \psi^{4} V_{02}^{2}-4 \psi^{2} V_{11}^{2}+4\left(-1+\psi^{2} V_{02}\right) V_{20}\right\}\right]}{4\left\{V_{11}^{2}-V_{02}\left(1+2 \psi^{2} V_{02}+V_{20}\right)\right\}} .
\end{aligned}
$$

3.6. Singh and Solanki [19]. Singh and Solanki [19] proposed a family of estimators as given below:

$$
\widehat{\bar{Y}}_{S S 1}=\left[\theta_{9} \bar{y}_{s t}\left\{\frac{\gamma\left(a_{s t} \bar{x}_{s t}+b_{s t}\right)+(1-\gamma)\left(a_{s t} \bar{X}+b_{s t}\right)}{\left(a_{s t} \bar{X}+b_{s t}\right)}\right\}^{\delta_{1}}+\theta_{10} \bar{y}_{s t}\left\{\frac{\left(a_{s t} \bar{X}+b_{s t}\right)}{\gamma\left(a_{s t} \bar{x}_{s t}+b_{s t}\right)+(1-\gamma)\left(a_{s t} \bar{X}+b_{s t}\right)}\right\}^{\delta_{2}}\right],
$$

where $\delta_{1}$ and $\delta_{2}$ are suitable scalars and $\theta_{9}$ and $\theta_{10}$ are the constants to be determined to make the MSE minimum. Assuming different values of $\delta_{1}, \delta_{2}$, and $\gamma$ proposed family $\widehat{\bar{Y}}_{S S 1}$ reduces to the ratio-type $\widehat{\bar{Y}}_{S S 1 R}$, product-type $\widehat{\bar{Y}}_{S S 1 P}$, and ratio-cum-product-type $\bar{Y}_{S S 1 R P}$ estimators.
For ratio-type, product-type, and ratio-cum-producttype estimators suitable values are $\left(\delta_{1}=0, \delta_{2}=1, \gamma=1\right),\left(\delta_{1}=\right.$ $\left.0, \delta_{2}=-1, \gamma=1\right)$, and $\left(\delta_{1}=1, \delta_{2}=1, \gamma=1\right)$, respectively.

For bias and MSE of $\bar{Y}_{S S 1}$, we consider the expressions given below:

$$
\begin{aligned}
& \operatorname{Bias}\left(\widehat{\bar{Y}}_{S S 1}\right) \cong \bar{Y}\left[\theta_{9}\left\{1+\gamma \delta_{1} \eta V_{11}+\frac{\delta_{1}\left(\delta_{1}-1\right)}{2} \gamma^{2} \eta^{2} V_{02}\right\}+\theta_{10}\left\{1-\gamma \delta_{2} \eta V_{11}+\frac{\delta_{2}\left(\delta_{2}+1\right)}{2} \gamma^{2} \eta^{2} V_{02}\right\}-1\right], \\
& \operatorname{MSE}\left(\widehat{\bar{Y}}_{S S 1}\right) \cong \bar{Y}^{2}\left[1+\theta_{9}^{2} A_{1}+\theta_{10}^{2} A_{2}+2 \theta_{9} \theta_{10} A_{3}-2 \theta_{9} A_{4}-2 \theta_{10} A_{5}\right],
\end{aligned}
$$

where

$$
\begin{aligned}
& A_{1}=\left[1+V_{20}+4 \gamma \delta_{1} \eta V_{11}+\delta_{1}\left(2 \delta_{1}-1\right) \gamma^{2} \eta^{2} V_{02}\right] \\
& A_{2}=\left[1+V_{20}-4 \gamma \delta_{2} \eta V_{11}+\delta_{2}\left(2 \delta_{2}+1\right) \gamma^{2} \eta^{2} V_{02}\right] \\
& A_{3}=\left[1+V_{20}+2 \gamma\left(\delta_{1}-\delta_{2}\right) \eta V_{11}+\left(\frac{\gamma^{2} \eta^{2}}{2}\right)\left(\delta_{1}-\delta_{2}\right)\left(\delta_{1}-\delta_{2}-1\right) V_{02}\right], \\
& A_{4}=\left[1+\gamma \delta_{1} \eta V_{11}+\frac{\delta_{1}\left(\delta_{1}-1\right)}{2} \gamma^{2} \eta^{2} V_{02}\right] \\
& A_{5}=\left[1-\gamma \delta_{2} \eta V_{11}+\frac{\delta_{2}\left(\delta_{2}+1\right)}{2} \gamma^{2} \eta^{2} V_{02}\right]
\end{aligned}
$$


The weights of $\theta_{9}$ and $\theta_{10}$ are determined as below:

$$
\begin{array}{r}
\theta_{9}=\frac{A_{2} A_{4}-A_{5} A_{3}}{A_{2} A_{1}-A_{3}^{2}}, \\
\theta_{10}=\frac{A_{1} A_{5}-A_{4} A_{3}}{A_{2} A_{1}-A_{3}^{2}} .
\end{array}
$$

Substituting the above weights in (31), we get the minimum MSE as given by

$$
\operatorname{MSE}_{\text {min }}\left(\widehat{\bar{Y}}_{S S 1}\right) \cong \bar{Y}^{2}\left[1-\frac{A_{2} A_{4}^{2}-2 A_{4} A_{3} A_{5}+A_{5}^{2} A_{1}}{A_{2} A_{1}-A_{3}^{2}}\right]
$$

3.7. Solanki and Singh [10]. Given below is the class of estimators suggested by Solanki and Singh [10]:

$$
\widehat{\bar{Y}}_{S S 2}=\left[\theta_{11} \bar{y}_{s t}\left[\frac{a_{s t} \bar{X}+b_{s t}}{\gamma\left(a_{s t} \bar{x}_{s t}+b_{s t}\right)+(1-\gamma)\left(a_{s t} \bar{X}+b_{s t}\right)}\right]^{\delta_{3}}+\theta_{12} \bar{y}_{s t} \exp \left[\frac{\delta_{4}\left\{\left(a_{s t} \bar{X}+b_{s t}\right)-\left(a_{s t} \bar{x}_{s t}+b_{s t}\right)\right\}}{\left(a_{s t} \bar{X}+b_{s t}\right)+\left(a_{s t} \bar{x}_{s t}+b_{s t}\right)}\right]\right],
$$

where $\theta_{11}$ and $\theta_{12}$ are the feasible weights to be found such that the MSE is minimal. Here, $\gamma=1 ; \delta_{3}$ and $\delta_{4}$ being the constants take values $(0,1,-1)$ for obtaining different estimators like

(i) Ratio-type exponential estimators $\hat{\bar{Y}}_{S S 2 R}$ for $\left(\delta_{3}=0, \delta_{4}=1\right)$

(ii) Product-type exponential estimators $\hat{\bar{Y}}_{S S 2 P}$ for $\left(\delta_{3}=0, \delta_{4}=-1\right)$

(iii) Ratio-ratio-type exponential estimators $\hat{\bar{Y}}_{S S 2 R R}$ for $\left(\delta_{3}=1, \delta_{4}=1\right)$ (iv) Product-product-type exponential estimators $\overline{\bar{Y}}_{S S 2 P P}$ for $\left(\delta_{3}=-1, \delta_{4}=-1\right)$

(v) Ratio-product-type exponential estimators $\hat{\bar{Y}}_{\text {SS2RP }}$ for $\left(\delta_{3}=1, \delta_{4}=-1\right)$

(vi) Product-ratio-type exponential estimators $\widehat{\bar{Y}}_{S S 2 P R}$ for $\left(\delta_{3}=-1, \delta_{4}=1\right)$

For bias and MSE of $\hat{\bar{Y}}_{S S 2}$, we consider the expressions given below:

$$
\begin{aligned}
& \operatorname{Bias}\left(\hat{\bar{Y}}_{S S 2}\right) \cong \bar{Y}\left[\theta_{11}\left\{1+\frac{\gamma \delta_{3} \eta}{2} V_{02}\left(\gamma \eta\left(\delta_{3}+1\right)-2 \frac{V_{11}}{V_{02}}\right)\right\}+\theta_{12}\left\{1+\frac{\delta_{4} \eta}{8} V_{02}\left(\eta\left(\delta_{4}+2\right)-4 \frac{V_{11}}{V_{02}}\right)\right\}-1\right], \\
& \operatorname{MSE}\left(\hat{\bar{Y}}_{S S 2}\right) \cong \bar{Y}^{2}\left[1+\theta_{11}^{2} B_{1}+\theta_{12}^{2} B_{2}+2 \theta_{11} \theta_{12} B_{3}-2 \theta_{11} B_{4}-2 \theta_{12} B_{5}\right],
\end{aligned}
$$

where

$$
\begin{aligned}
& B_{1}=\left[1+V_{20}+\gamma^{2} \eta^{2}\left(2 \delta_{3}^{2}+\delta_{3}\right) V_{02}-4 \gamma \delta_{3} \eta V_{11}\right], \\
& B_{2}=\left[1+V_{20}+\frac{\eta^{2}\left(\delta_{4}^{2}+\delta_{4}\right)}{2} V_{02}-2 \delta_{4} \eta V_{11}\right], \\
& B_{3}=\left[1+V_{20}+\left(\frac{\eta^{2}\left[\left(2 \gamma \delta_{3}+\delta_{4}\right)^{2}+2\left(2 \gamma^{2} \delta_{3}+\delta_{4}\right)\right]}{8}\right) V_{02}-\eta\left(2 \gamma \delta_{3}+\delta_{4}\right) V_{11}\right], \\
& B_{4}=\left[1+\frac{\gamma^{2} \eta^{2}\left(\delta_{3}^{2}+\delta_{3}\right)}{2} V_{02}-\gamma \delta_{3} \eta V_{11}\right], \\
& B_{5}=\left[1+\frac{\eta^{2}\left(\delta_{4}^{2}+2 \delta_{4}\right)}{8} V_{02}-\frac{\delta_{4} \eta}{2} V_{11}\right] .
\end{aligned}
$$


The suitable weights of $\theta_{11}$ and $\theta_{12}$ are as below:

$$
\begin{gathered}
\theta_{11}=\frac{B_{2} B_{4}-B_{5} B_{3}}{B_{2} B_{1}-B_{3}^{2}}, \\
\theta_{12}=\frac{B_{1} B_{5}-B_{4} B_{3}}{B_{2} B_{1}-B_{3}^{2}} .
\end{gathered}
$$

Substituting these suitable weights in (37), we have the minimum MSE as given by

$$
\operatorname{MSE}_{\min }\left(\widehat{\bar{Y}}_{S S 2}\right) \cong \bar{Y}^{2}\left[1-\frac{B_{2} B_{4}^{2}-2 B_{4} B_{3} B_{5}+B_{5}^{2} B_{1}}{B_{2} B_{1}-B_{3}^{2}}\right]
$$

3.8. Solanki and Singh [12]. Recently, Solanki and Singh [12] defined an improved estimation given as

$$
\widehat{\bar{Y}}_{S S 3}=\left[\theta_{13} \bar{y}_{s t}\left(\frac{\bar{X}_{s t}^{*}}{\bar{x}_{s t}^{*}}\right)^{\delta_{5}} \exp \left\{\frac{\delta_{6}\left(\bar{X}_{s t}^{*}-\bar{x}_{s t}^{*}\right)}{\left(\bar{X}_{s t}^{*}+\bar{x}_{s t}^{*}\right)}\right\}+\theta_{14} \bar{y}_{s t}\left(\frac{\bar{x}_{s t}^{*}}{\bar{X}_{s t}^{*}}\right)^{\delta_{7}} \exp \left\{\frac{\delta_{8}\left(\bar{x}_{s t}^{*}-\bar{X}_{s t}^{*}\right)}{\left(\bar{X}_{s t}^{*}+\bar{x}_{s t}^{*}\right)}\right\}\right],
$$

where $\theta_{13}$ and $\theta_{14}$ are the suitably chosen weights to get minimum MSE. $\quad \bar{X}_{s t}^{*}=\sum_{h=1}^{L} W_{h}\left(a_{h} \bar{X}_{h}+b_{h}\right)$ and $\bar{x}_{s t}^{*}=\sum_{h=1}^{L} W_{h}\left(a_{h} \bar{x}_{h}+b_{h}\right), a_{h}(\neq 0), b_{h}$, are real number to parameters related to auxiliary variate $x$. Here, $\delta_{5}, \delta_{6}, \delta_{7}$, and $\delta_{8}$ being the constants take values $(-1,0,1)$ for obtaining different classes of estimators $\bar{Y}_{S S 3 i}$ :

(i) $\widehat{\bar{Y}}_{S S 31}$, for $\delta_{5}=-1, \delta_{6}=1, \delta_{7}=1$, and $\delta_{8}=1$ (ii) $\widehat{\bar{Y}}_{S S 32}$, for $\delta_{5}=-1, \delta_{6}=1, \delta_{7}=1$, and $\delta_{8}=0$

(iii) $\widehat{\bar{Y}}_{S S 33}$, for $\delta_{5}=+1, \delta_{6}=1, \delta_{7}=1$, and $\delta_{8}=1$

For bias and MSE of $\hat{\bar{Y}}_{S S 3}$, we consider the expressions given below:

$$
\operatorname{Bias}\left(\widehat{\bar{Y}}_{S S 3}\right) \cong\left[\begin{array}{c}
\left(\theta_{13}+\theta_{14}-1\right) \bar{Y}-\frac{t_{1} \theta_{13}}{2 \bar{X}_{s t}^{*}} \sum_{h=1}^{L} W_{h}^{2} \phi_{h} a_{h} s_{x h}^{2}\left\{t_{3}+\frac{\left(t_{1}-2\right)}{4} a_{h} R^{*}\right\} \\
+\frac{t_{2} \theta_{14}}{2 \bar{X}_{s t}^{*}} \sum_{h=1}^{L} W_{h}^{2} \phi_{h} a_{h} S_{x h}^{2}\left\{t_{3}-\frac{\left(t_{2}-2\right)}{4} a_{h} R^{*}\right\}
\end{array}\right],
$$

where $t_{1}=\left(2 \delta_{5}+\delta_{6}\right), t_{2}=\left(2 \delta_{7}+\delta_{8}\right)$, and $t_{3}=S_{y x h} / S_{x h}^{2}$ and $R^{*}=\left(\bar{Y} / \bar{X}_{s t}^{*}\right)$ :

$$
\operatorname{MSE}\left(\widehat{\bar{Y}}_{S S 3}\right) \cong \bar{Y}^{2}\left[1+\theta_{13}^{2} C_{1}+\theta_{14}^{2} C_{2}+2 \theta_{13} \theta_{14} C_{3}-2 \theta_{13} C_{4}-2 \theta_{14} C_{5}\right]
$$


where

$$
\begin{aligned}
& C_{1}=1+\frac{1}{\bar{Y}^{2}} \sum_{h=1}^{L} W_{h}^{2} \phi_{h}\left\{S_{y h}^{2}-2 t_{1} a_{h} R^{*} S_{y x h}+\frac{t_{1}\left(t_{1}+1\right)}{2} a_{h}^{2} R^{* 2} S_{x h}^{2}\right\}, \\
& C_{2}=1+\frac{1}{\bar{Y}^{2}} \sum_{h=1}^{L} W_{h}^{2} \phi_{h}\left\{S_{y h}^{2}+2 t_{2} a_{h} R^{*} S_{y x h}+\frac{t_{2}\left(t_{2}-1\right)}{2} a_{h}^{2} R^{* 2} S_{x h}^{2}\right\}, \\
& C_{3}=1+\frac{1}{\bar{Y}^{2}} \sum_{h=1}^{L} W_{h}^{2} \phi_{h}\left\{S_{y h}^{2}+\left(t_{2}-t_{1}\right) a_{h} R^{*} S_{y x h}+\frac{\left(t_{2}-t_{1}\right)\left(t_{2}-t_{1}-2\right)}{8} a_{h}^{2} R^{* 2} S_{x h}^{2}\right\}, \\
& C_{4}=1-\frac{t_{1}}{2 \bar{Y}} \sum_{h=1}^{L} W_{h}^{2} \phi_{h}\left\{R^{*} a_{h} S_{y x h}-\frac{\left(t_{1}+2\right)}{4} a_{h}^{2} R^{* 2} S_{x h}^{2}\right\}, \\
& C_{5}=1+\frac{t_{2}}{2 \bar{Y}} \sum_{h=1}^{L} W_{h}^{2} \phi_{h}\left\{R^{*} a_{h} S_{y x h}+\frac{\left(t_{2}-2\right)}{4} a_{h}^{2} R^{* 2} S_{x h}^{2}\right\} .
\end{aligned}
$$

Given below are the weights of $\theta_{13}$ and $\theta_{14}$ for minimizing the MSE:

$$
\begin{aligned}
& \theta_{13}=\frac{C_{2} C_{4}-C_{5} C_{3}}{C_{2} C_{1}-C_{3}^{2}}, \\
& \theta_{14}=\frac{C_{1} C_{5}-C_{4} C_{3}}{C_{2} C_{1}-C_{3}^{2}} .
\end{aligned}
$$

Thus, the minimum MSE by putting the above values of $\theta_{13}$ and $\theta_{14}$ in (43) is given by

$$
\operatorname{MSE}_{\min }\left(\hat{\bar{Y}}_{S S 3}\right) \cong \bar{Y}^{2}\left[1-\frac{C_{1} C_{5}^{2}-2 C_{4} C_{3} C_{5}+C_{4}^{2} C_{2}}{C_{2} C_{1}-C_{3}^{2}}\right] .
$$

\section{Suggested Family of Estimators}

Following the lines of Shabbir et al. [20], a generalized estimator for the estimation of population mean is proposed using some traditional and nontraditional measures of an auxiliary variable. For more details of these nontraditional measures, see the works of Hettmansperger and McKean [21], Wang et al. [22], and Irfan et al. [23-26]:

$$
\widehat{\bar{Y}}_{P}=\left[\widehat{\bar{Y}}_{B T, \mathrm{Avg}}+\theta_{15}\left(\bar{X}-\bar{x}_{s t}\right)+\theta_{16} \bar{y}_{s t}\right] \exp \left(\frac{a_{s t} \bar{X}+b_{s t}}{a_{s t} \bar{x}_{s t}+b_{s t}}-1\right),
$$

where $\theta_{15}$ and $\theta_{16}$ are the suitably chosen weights and $a_{s t}(\neq 0)$ and $b_{s t}$ are either real numbers or functions of known parameters of the auxiliary variable such as standard deviation $S_{x s t}$, coefficient of skewness $\beta_{1 x s t}$, coefficient of kurtosis $\beta_{2 x s t}$, coefficient of variation $C_{x s t}$, coefficient of correlation $\rho_{s t}$, quartile deviation $Q D_{x s t}$, midrange $M R_{x s t}$, interquartile range $Q R_{x s t}$, quartile average $Q A_{x s t}$, tri-mean $T M_{x s t}$, and Hodge-Lehmann estimator $H L_{x s t}$.
Remark 1. Many more estimators can be generated by placing different available parameters of auxiliary variable in place of $a_{s t}$ and $b_{s t}$. Some of them are presented in Table 1 .

Using (1), the suggested class of estimators $\hat{\bar{Y}}_{P}$ can be rewritten as

$$
\begin{aligned}
\hat{\bar{Y}}_{P}= & {\left[\frac{\bar{Y}\left(1+\zeta_{0}\right)}{2}\left\{\exp \left(-\frac{\zeta_{1}}{2}\left(1+\frac{\zeta_{1}}{2}\right)^{-1}\right)+\exp \left(\frac{\zeta_{1}}{2}\left(1+\frac{\zeta_{1}}{2}\right)^{-1}\right)\right\}-\theta_{15} \bar{X} \zeta_{1}+\theta_{16} \bar{Y}\left(1+\zeta_{0}\right)\right] } \\
& \cdot \exp \left(-\eta \zeta_{1}\left(1+\eta \zeta_{1}\right)^{-1}\right) \times\left\{\frac{1}{2}\left(2+2 \eta \zeta_{1}+\eta^{2} \zeta_{1}^{2}\right)\left(1+\eta \zeta_{1}\right)^{-1}\right\}^{2} .
\end{aligned}
$$

As defined earlier, $\eta=a_{s t} \bar{X} / a_{s t} \bar{X}+b_{s t}$.
Expanding the right-hand side of (48), up to first order of approximation and subtracting $\bar{Y}$ from both sides, we obtain 
TABLE 1: Some members of the proposed family.

\begin{tabular}{|c|c|c|c|}
\hline Estimator & $\hat{\bar{Y}}_{P}^{(i)}$ & $a_{s t}$ & $b_{s t}$ \\
\hline 1 & $\hat{\bar{Y}}_{P}^{(1)}$ & 1 & 0 \\
\hline 2 & $\hat{\bar{Y}}_{P}^{(2)}$ & 1 & $\rho_{s t}=\sum_{h=1}^{L} W_{h} \rho_{h}$ \\
\hline 3 & $\hat{\bar{Y}}_{P}^{(3)}$ & 1 & $C_{x s t}=\sum_{h=1}^{L} W_{h} C_{x h}$ \\
\hline 4 & $\hat{\bar{Y}}_{P}^{(4)}$ & $C_{x s t}=\sum_{h=1}^{L} W_{h} C_{x h}$ & $\beta_{2 x s t}=\sum_{h=1}^{L} W_{h} \beta_{2 x h}$ \\
\hline 5 & $\hat{\bar{Y}}_{P}^{(5)}$ & $\beta_{1 x s t}=\sum_{h=1}^{L} W_{h} \beta_{1 x h}$ & $C_{x s t}=\sum_{h=1}^{L} W_{h} C_{x h}$ \\
\hline 6 & $\hat{\bar{Y}}_{P}^{(6)}$ & $\beta_{1 x s t}=\sum_{h=1}^{L} W_{h} \beta_{1 x h}$ & $\rho_{s t}=\sum_{h=1}^{L} W_{h} \rho_{h}$ \\
\hline 7 & $\hat{\bar{Y}}_{P}^{(7)}$ & 1 & $\beta_{2 x s t}=\sum_{h=1}^{L} W_{h} \beta_{2 x h}$ \\
\hline 8 & $\hat{\bar{Y}}_{P}^{(8)}$ & 1 & $\beta_{1 x s t}=\sum_{h=1}^{L} W_{h} \beta_{1 x h}$ \\
\hline 9 & $\hat{\bar{Y}}_{P}^{(9)}$ & $T M_{x s t}=\sum_{h=1}^{L} W_{h} T M_{x h}$ & 1 \\
\hline 10 & $\hat{\bar{Y}}_{P}^{(10)}$ & $T M_{x s t}=\sum_{h=1}^{L} W_{h} T M_{x h}$ & $Q R_{x s t}=\sum_{h=1}^{L} W_{h} Q R_{x h}$ \\
\hline 11 & $\hat{\bar{Y}}_{P}^{(11)}$ & $Q A_{x s t}=\sum_{h=1}^{L} W_{h} Q A_{x h}$ & $S_{x s t}=\sum_{h=1}^{L} W_{h} S_{x h}$ \\
\hline 12 & $\hat{\bar{Y}}_{P}^{(12)}$ & $H L_{x s t}=\sum_{h=1}^{L} W_{h} H L_{x h}$ & $\rho_{s t}=\sum_{h=1}^{L} W_{h} \rho_{h}$ \\
\hline 13 & $\hat{\bar{Y}}_{P}^{(13)}$ & $H L_{x s t}=\sum_{h=1}^{L} W_{h} H L_{x h}$ & $Q R_{x s t}=\sum_{h=1}^{L} W_{h} Q R_{x h}$ \\
\hline 14 & $\hat{\bar{Y}}_{P}^{(14)}$ & $M R_{x s t}=\sum_{h=1}^{L} W_{h} M R_{x h}$ & $Q R_{x s t}=\sum_{h=1}^{L} W_{h} Q R_{x h}$ \\
\hline 15 & $\widehat{\widehat{Y}}_{P}^{(13)}$ & $M R_{x s t}=\sum_{h=1}^{L} W_{h} M R_{x h}$ & $Q D_{x s t}=\sum_{h=1}^{L} W_{h} Q D_{x h}$ \\
\hline
\end{tabular}

Up to first order of approximation, the bias and the MSE are given by

$$
\begin{aligned}
\operatorname{Bias}\left(\hat{\bar{Y}}_{P}\right) \cong & \bar{Y}\left[V_{02}\left(\frac{1+20 \eta^{2}}{8}\right)-\eta V_{11}\right]+\bar{X} \eta \theta_{15} V_{02}+\theta_{16}\left(\bar{Y}+\frac{5 \bar{Y} \eta^{2} V_{02}}{2}-\bar{Y} \eta V_{11}\right) \\
\operatorname{MSE}\left(\hat{\bar{Y}}_{P}\right) \cong & \bar{Y}^{2} \eta^{2} V_{02}+\bar{Y}^{2} V_{20}-2 \bar{Y}^{2} \eta V_{11}+\bar{X}^{2} \theta_{15}^{2} V_{02}+2 \bar{X} \bar{Y} \eta \theta_{15} V_{02}-2 \bar{X} \bar{Y} \theta_{15} V_{11} \\
& +\bar{Y} \theta_{16}\left\{4 \bar{X} \eta \theta_{15} V_{02}-2 \bar{X} \theta_{15} V_{11}+7 \eta^{2} \bar{Y} V_{02}-6 \eta \bar{Y} V_{11}+2 \bar{Y} V_{20}+\frac{1}{4} \bar{Y} V_{02}\right\}+\bar{Y}^{2} \theta_{16}^{2}\left\{1+6 \eta^{2} V_{02}-4 \eta V_{11}+V_{20}\right\} .
\end{aligned}
$$

By minimizing (51), suitable weights of $\theta_{15}$ and $\theta_{16}$ are obtained as below:

$$
\begin{aligned}
\theta_{15} & =\frac{\bar{Y}\left\{2 \eta V_{02}^{2}\left(\eta^{2}+1 / 4\right)+2 V_{11}\left(1-\eta V_{11}\right)-\eta V_{02}\left(2-\eta V_{11}-2 V_{20}\right)-1 / 4 V_{11} V_{02}\right\}}{2 \bar{X}\left\{-V_{11}^{2}+V_{02}\left(1+2 \eta^{2} V_{02}+V_{20}\right)\right\}}, \\
\theta_{16} & =\frac{2 V_{11}^{2}-V_{02}\left(3 \eta^{2} V_{02}+2 V_{20}+V_{02} / 4\right)}{2\left\{-V_{11}^{2}+V_{02}\left(1+2 \eta^{2} V_{02}+V_{20}\right)\right\}} .
\end{aligned}
$$


Putting the optimal weights of $\theta_{15}$ and $\theta_{16}$ in (51), we have the minimum MSE given by

$$
\operatorname{MSE}_{\min }\left(\hat{\bar{Y}}_{P}\right) \cong \frac{\bar{Y}^{2}\left[4 V_{11}^{2}+V_{02}\left\{3 \eta^{2} V_{02}^{2}\left(3 \eta^{2}+1 / 2\right)-V_{11}^{2}\left(1+4 \eta^{2}\right)+1 / 16 V_{02}^{2}+4\left(-1+V_{02}\left(\eta^{2}+1 / 4\right)\right) V_{20}\right\}\right]}{4\left\{V_{11}^{2}-V_{02}\left(1+2 \eta^{2} V_{02}+V_{20}\right)\right\}} .
$$

\section{Application to a Dataset}

To examine the performance of the proposed class of estimators, we considered a real data of Turkey (2007) used by Koyuncu and Kadilar [3] given in Table 2. In this data, let $y$ be the number of teachers (study variable) and $x$ be the number of students (auxiliary variable) which are recorded for primary and secondary schools at 6 regions for $N=923$ districts. A total sample of size $n=180$ is selected through Neyman allocation from 6 strata. (source: Ministry of Education, Republic of Turkey).

We have computed $\operatorname{MSE}_{\min }\left(\hat{\bar{Y}}_{K}\right), \operatorname{MSE}_{\min }\left(\hat{\bar{Y}}_{K K}\right)$, $\operatorname{MSE}_{\min }\left(\hat{\bar{Y}}_{S G}\right), \operatorname{MSE}_{\min }\left(\hat{\bar{Y}}_{H S 1}\right), \quad \operatorname{MSE}_{\min }\left(\hat{\bar{Y}}_{H S 2}\right), \quad \mathrm{MSE}_{\min }$ $\left(\widehat{\bar{Y}}_{S S 1}\right), \operatorname{MSE}_{\min }\left(\widehat{\bar{Y}}_{S S 2}\right), \operatorname{MSE}_{\min }\left(\widehat{\bar{Y}}_{S S 3}\right)$, and $\operatorname{MSE}_{\min }\left(\hat{\bar{Y}}_{P}\right)$ for the population dataset given in Table 2 and are reported in Tables 3 and 4 .

\subsection{Important Findings}

(i) The values of MSE of usual unbiased, combined ratio, and combined regression estimators in stratified random sampling are computed as given below:

$$
\begin{aligned}
\operatorname{MSE}\left(\hat{\bar{Y}}_{s t}\right) & =2229.2662, \\
\operatorname{MSE}\left(\widehat{\bar{Y}}_{C R}\right) & =216.4168, \\
\operatorname{MSE}\left(\hat{\bar{Y}}_{\text {CReg }}\right) & =194.2832 .
\end{aligned}
$$

(ii) It is obvious from Table 3 that MSEs of Koyuncu and Kadilar [3] class of estimators $\overline{\bar{Y}}_{K}$ round about the value of $\operatorname{MSE}\left(\overline{\bar{Y}}_{C R}\right)$.

(iii) value of $\operatorname{MSE}\left(\hat{\bar{Y}}_{C \text { Reg }}\right)$ is less than $\operatorname{MSE}\left(\hat{\bar{Y}}_{C R}\right)$ and $\operatorname{MSE}_{\min }\left(\overline{\bar{Y}}_{K}\right)$.

(iv) It is observed from Tables 3 and 4 that the MSEs of Koyuncu and Kadilar [4] class of estimators $\widehat{\bar{Y}}_{K K}$, Shabbir and Gupta [8] class of estimators $\widehat{\bar{Y}}_{S G}$, Haq and Shabbir [9] classes of estimators $\widehat{\bar{Y}}_{H S i}, i=1,2$, and Singh and Solanki $[10,12]$ classes of estimators $\overline{\bar{Y}}_{S S j}, j=1,2,3$, are less than the MSE of combined regression estimator $\bar{Y}_{C \text { Reg }}$.

(v) Again, from Tables 3 and 4, it is valuable to mention that the proposed family of estimators $\widehat{\bar{Y}}_{P}$ have the least MSEs as compared to all other classes of estimators against different values of $a_{s t}$ and $b_{s t}$.

\section{Simulation Study}

In this section, we carried out a simulation study using $\mathrm{R}$ statistical software to evaluate the behavior of proposed estimators $\hat{\bar{Y}}_{P}^{(i)}$ in comparison with $\widehat{\bar{Y}}_{s t}, \widehat{\bar{Y}}_{C R}, \hat{\bar{Y}}_{C R e g}, \widehat{\bar{Y}}_{K}$, $\widehat{\bar{Y}}_{K K}, \widehat{\bar{Y}}_{S G}, \widehat{\bar{Y}}_{H S 1}, \widehat{\bar{Y}}_{H S 2}, \widehat{\bar{Y}}_{S S 1}, \widehat{\bar{Y}}_{S S 2}$, and $\widehat{\bar{Y}}_{S S 3}$. Real population presented in Table 3 is used for the simulation study. Three different sample sizes $n=180,250$, and 350 are taken from this population on the basis of proportional allocation.

The following steps summarize the procedure of finding the average MSE of an estimator.

Step 1: select a bivariate stratified sample of size $n$ using simple random sampling without replacement from the bivariate stratified normal population

Step 2: use sample data from Step 1 to find the MSE of all the estimators under the study

Step 3: repeat Step 1 and Step 2 10,000 times and obtain 10,000 values for MSEs

Step 4: Average of 10,000 values obtained in Step 3 are the MSE of each estimator

6.1. Findings. MSEs of $\hat{\bar{Y}}_{s t}, \widehat{\bar{Y}}_{C R}$, and $\widehat{\bar{Y}}_{C R e g}$ for different sample sizes are under Table 5.

MSEs of the other estimators under the study are presented in Tables 6-11, and the following important considerations are made from them.

(i) $\mathrm{It}_{(i)}$ is quite obvious that the MSE values of $\widehat{\bar{Y}}_{P}^{(i)}, i=1,2,3, \ldots, 15$, as compared to the MSEs of all other estimators are minimum under different sample sizes taken from the population. It proves that all the proposed estimators are more efficient.

(ii) It is also shown that, by increasing the sample size selected from the population, the MSEs decrease.

\section{Discussion}

In this study, we proposed a new optimal family of estimators for estimating population mean under stratified random sampling. Bias, MSE, and minimum MSE of this family of estimators are derived up to first degree of approximation. The proposed family is compared with some well-known estimators/classes of estimators under stratified random sampling such as the works of Koyuncu and Kadilar [3, 4], Shabbir and Gupta [8], Haq and Shabbir [9], Singh and Solanki [19], and Solanki and Singh [10, 12]. It is numerically inferred that the proposed family behaves optimal 
TABle 2: Data statistics.

\begin{tabular}{|c|c|c|c|c|c|c|}
\hline \multirow{2}{*}{ Values } & \multicolumn{6}{|c|}{$h^{\text {th }}$ stratum } \\
\hline & 1 & 2 & 3 & 4 & 5 & 6 \\
\hline$N_{h}$ & 127 & 117 & 103 & 170 & 205 & 201 \\
\hline$n_{h}$ & 31 & 21 & 29 & 38 & 22 & 39 \\
\hline $\bar{Y}_{h}$ & 703.74 & 413.00 & 573.17 & 424.66 & 267.03 & 393.84 \\
\hline $\bar{X}_{h}^{n}$ & 20804.59 & 9211.79 & 14309.3 & 9478.85 & 5569.95 & 12997.59 \\
\hline$S_{y h}$ & 883.835 & 644.922 & 1033.467 & 810.585 & 403.654 & 711.723 \\
\hline$S_{x h}$ & 30486.751 & 15180.769 & 27549.697 & 18218.931 & 8497.776 & 23094.141 \\
\hline$S_{y x h}$ & 25237153.52 & 9747942.85 & 28294397.04 & 14523885.53 & 3393591.75 & 15864573.97 \\
\hline$C_{x h}$ & 1.465 & 1.648 & 1.925 & 1.922 & 1.526 & 1.777 \\
\hline$\rho_{h}$ & 0.936 & 0.996 & 0.994 & 0.983 & 0.989 & 0.965 \\
\hline$\beta_{2 h(x)}$ & 4.593 & 18.543 & 15.446 & 10.162 & 21.947 & 23.114 \\
\hline$\beta_{1 h(x)}$ & 2.164 & 3.867 & 3.748 & 3.121 & 4.084 & 4.411 \\
\hline$Q D_{x h}$ & 12736.8 & 3023.5 & 5698.75 & 2742.25 & 2025.5 & 5545.5 \\
\hline$M R_{x h}$ & 77193.5 & 54644 & 85780.5 & 56386.5 & 34057 & 89530.5 \\
\hline$Q R_{x h}$ & 25473.5 & 6047 & 11397.5 & 5484.5 & 4051 & 11091 \\
\hline$Q A_{x h}$ & 15489.3 & 5104.5 & 7704.25 & 3899.25 & 3468.5 & 8105.5 \\
\hline$T M_{x h}$ & 10853.1 & 4705.75 & 5994.13 & 3321.38 & 3137.75 & 7116.25 \\
\hline$H L_{x h}$ & 7913.75 & 4883.25 & 7124.5 & 3899.5 & 3173.25 & 8074 \\
\hline
\end{tabular}

TABle 3: Minimum MSEs of different estimators based on real data.

\begin{tabular}{|c|c|c|c|c|c|c|c|c|c|c|}
\hline$a_{s t}$ & $b_{s t}$ & $\hat{\bar{Y}}_{K}$ & $\hat{\bar{Y}}_{K K}$ & $\hat{\bar{Y}}_{S G}$ & $\widehat{\bar{Y}}_{H S 1}$ & $\widehat{\bar{Y}}_{H S 2}$ & $\hat{\bar{Y}}_{S S 1 R}$ & $\hat{\bar{Y}}_{S S 1 P}$ & $\hat{\bar{Y}}_{S S 1 R P}$ & $\hat{\bar{Y}}_{P}$ \\
\hline & 0 & & & & & & & 175.9254 & & 102.9573 \\
\hline & $\sum_{h=1}^{L} W_{h} \rho_{h}$ & 216.3768 & 194.0826 & 18.002 & 183.4853 & 115.8949 & 193.4192 & 175.9254 & 192.8484 & 102.9843 \\
\hline 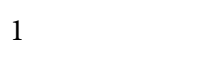 & $\sum_{h=1}^{L} W_{h} C_{x h}$ & 6.3470 & 94.0826 & 194.0852 & 183.4877 & 115.9136 & 193.4198 & 175.9254 & 192.8496 & 103.0045 \\
\hline$\sum_{h=1}^{L} W_{h} C_{x h}$ & $\sum_{h=1}^{L} W_{h} \beta_{2 x h}$ & 216.0228 & 194.0826 & 194.0852 & 1835136 & 116.1178 & 193.4264 & 175.9254 & 192.8633 & 103.2247 \\
\hline$\sum_{h=1}^{L} W_{h} \beta_{1 x h}$ & $\sum_{h=1}^{L} W_{h} C_{x h}$ & 16.3976 & 194.0826 & 194.0852 & 183.4836 & 115.8818 & 193.4187 & 175.9254 & 192.8475 & 102.9702 \\
\hline$\sum_{h=1}^{L} W_{h} \beta_{1 x h}$ & $\sum_{h=1}^{L} W_{h} \rho_{h}$ & 216.4058 & 194.0826 & 194.0852 & 183.4830 & 115.8766 & 193.4186 & 175.9254 & 192.8472 & 1029647 \\
\hline 1 & $\sum_{h=1}^{L} W_{h} \beta_{2 x h}$ & 215.7475 & 194.0826 & 194.0852 & 183.5357 & 116.2921 & 193.4320 & 175.9254 & 192.8749 & 103.4126 \\
\hline & $\sum_{h=1}^{L} W_{h} \beta_{1 x h}$ & 216.2676 & 194.0826 & 194.0 & 183.4940 & 115.9635 & 193.4 & 175.5 & 192.8530 & 103.0583 \\
\hline$\sum_{h=1}^{L} W_{h} T M_{x h}$ & 1 & 216.4168 & 194.0826 & 194.0852 & 183.4821 & 115.8697 & 193.4183 & 175.9254 & 192.8467 & 1029573 \\
\hline$\sum_{h=1}^{L} W_{h} T M_{x h}$ & $\sum_{h=1}^{L} W_{h} Q R_{x h}$ & 216.3449 & 194.0826 & 194.0852 & 183.4878 & 115.9149 & 193.4198 & 175.9254 & 192.8497 & 103.0059 \\
\hline$\sum_{h=1}^{L} W_{h} Q A_{x h}$ & $\sum_{h=1}^{L} W_{h} S_{x h}$ & 216.3012 & 194.0826 & 194.0852 & & & & 175.9254 & 192.8516 & 103.0355 \\
\hline$\sum_{h=1}^{L} W_{h} H L_{x h}$ & $\sum_{h=1}^{L} W_{h} \rho_{h}$ & 216.4168 & 194.0826 & 194.0852 & 183.4821 & 115.8697 & 193.4183 & 175.9254 & 192.8467 & 102.9573 \\
\hline$\sum_{h=1}^{L} W_{h} H L_{x h}$ & $\sum_{h=1}^{L} W_{h} Q R_{x h}$ & 216.3457 & 194.0826 & 194.0852 & 183.4878 & 115.9144 & 193.4198 & 175.9254 & 192.8497 & 103.0054 \\
\hline$\sum_{h=1}^{L} W_{h} M R_{x h}$ & $\sum_{h=1}^{L} W_{h} Q R_{x h}$ & 216.4105 & 194.0826 & 194.0852 & 183.4826 & 115.8737 & 193.4185 & 175.9254 & 192.8470 & 102.9615 \\
\hline$\sum_{h=1}^{L} W_{h} M R_{x h}$ & $\sum_{h=1}^{L} W_{h} Q D_{x h}$ & 216.4136 & 194.0826 & 194.0852 & 183.4823 & 115.8717 & 193.4184 & 175.9254 & 192.8468 & 102.9594 \\
\hline
\end{tabular}

Bold values indicate minimum MSE.

TABLE 4: Minimum MSEs of different estimators based on real data.

\begin{tabular}{|c|c|c|c|c|c|c|c|c|c|c|c|}
\hline$a_{s t}$ & $b_{s t}$ & $\widehat{\bar{Y}}_{S S 2 R}$ & $\widehat{\bar{Y}}_{S S 2 P}$ & $\widehat{\bar{Y}}_{S S 2 R R}$ & $\widehat{\bar{Y}}_{S S 2 P P}$ & $\widehat{\bar{Y}}_{S S 2 R P}$ & $\widehat{\bar{Y}}_{S S 2 P R}$ & $\widehat{\bar{Y}}_{S S 31}$ & $\widehat{\bar{Y}}_{S S 32}$ & $\widehat{\bar{Y}}_{S S 33}$ & $\widehat{\bar{Y}}_{P}$ \\
\hline 1 & 0 & 194.1562 & 185.5207 & 193.6484 & 143.7752 & 193.6484 & 191.9754 & 108.8591 & 143.7752 & 172.0985 & 102.9573 \\
\hline 1 & $\sum_{h=1}^{L} W_{h} \rho_{h}$ & 194.1560 & 185.5200 & 193.6489 & 143.7805 & 193.6489 & 191.9743 & 108.8704 & 143.7804 & 172.1103 & 102.9843 \\
\hline 1 & $\sum_{h=1}^{L} W_{h} C_{x h}$ & 194.1558 & 185.5195 & 193.6492 & 143.7844 & 193.6492 & 191.9735 & 108.8793 & 143.7844 & 172.1196 & 103.0045 \\
\hline$\sum_{h=1}^{L} W_{h} C_{x h}$ & $\sum_{h=1}^{L} W_{h} \beta_{2 x h}$ & 194.1539 & 185.5141 & 193.6532 & 143.8273 & 193.6532 & 191.9647 & 107.0285 & 132.7917 & 161.5885 & 103.2247 \\
\hline$\sum_{h=1}^{L} W_{h} \beta_{1 x h}$ & $\sum_{h=1}^{L} W_{h} C_{x h}$ & 194.1561 & 185.5203 & 193.6486 & 143.7777 & 193.1521 & 191.9749 & 178.5658 & 214.5583 & 231.1787 & 102.9702 \\
\hline$\sum_{h=1}^{L} W_{h} \beta_{1 x h}$ & $\sum_{h=1}^{L} W_{h} \rho_{h}$ & 194.1562 & 185.5205 & 193.6485 & 143.7767 & 193.1515 & 191.9751 & 178.5631 & 214.5571 & 231.1754 & 102.9647 \\
\hline 1 & $\sum_{h=1}^{L} W_{h} \beta_{2 x h}$ & 194.1522 & 185.5095 & 193.6566 & 143.8638 & 193.6566 & 191.9571 & 109.0538 & 143.8638 & 172.3019 & 103.4126 \\
\hline 1 & $\sum_{h=1}^{L} W_{h} \beta_{1 x h}$ & 194.1554 & 185.5182 & 193.6502 & 143.7949 & 193.1561 & 191.9714 & 108.9023 & 143.7949 & 172.1437 & 103.0583 \\
\hline
\end{tabular}


TABLE 4: Continued.

\begin{tabular}{|c|c|c|c|c|c|c|c|c|c|c|c|}
\hline$a_{s t}$ & $b_{s t}$ & $\hat{\bar{Y}}_{S S 2 R}$ & $\hat{\bar{Y}}_{S S 2 P}$ & $\widehat{\bar{Y}}_{S S 2 R R}$ & $\widehat{\bar{Y}}_{S S 2 P P}$ & $\hat{\bar{Y}}_{S S 2 R P}$ & $\widehat{\bar{Y}}_{S S 2 P R}$ & $\hat{\bar{Y}}_{S S 31}$ & $\widehat{\bar{Y}}_{S S 32}$ & $\widehat{\bar{Y}}_{S S 33}$ & $\widehat{\bar{Y}}_{P}$ \\
\hline$\sum_{h=1}^{L} W_{h} T M_{x h}$ & 1 & 194.1562 & 185.5207 & 193.6484 & 143.7752 & 193.1515 & 191.9754 & 676.9615 & 703.3570 & 656.1403 & 102.9573 \\
\hline$\sum_{h=1}^{L} W_{h} T M_{x h}$ & $\sum_{h=1}^{L} W_{h} Q R_{x h}$ & 194.1558 & 185.5195 & 193.6493 & 143.7847 & 193.1537 & 191.9735 & 676.9765 & 703.3632 & 656.1786 & 103.0059 \\
\hline$\sum_{h=1}^{L} W_{h} Q A_{x h}$ & $\sum_{h=1}^{L} W_{h} S_{x h}$ & 194.1556 & 185.5187 & 193.6498 & 143.7904 & 193.155 & 191.9723 & 820.1003 & 844.5858 & 772.9658 & 103.0355 \\
\hline$\sum_{h=1}^{L} W_{h} H L_{x h}$ & $\sum_{h=1}^{L} W_{h} \rho_{h}$ & 194.1562 & 185.5207 & 193.6484 & 143.7752 & 193.1515 & 191.9754 & 476.1121 & 506.1138 & 486.5211 & 102.9573 \\
\hline$\sum_{h=1}^{L} W_{h} H L_{x h}$ & $\sum_{h=1}^{L} W_{h} Q R_{x h}$ & 194.1558 & 185.5195 & 193.6493 & 143.7846 & 193.1537 & 191.9735 & 476.1289 & 506.1211 & 486.5537 & 103.0054 \\
\hline$\sum_{h=1}^{L} W_{h} M R_{x h}$ & $\sum_{h=1}^{L} W_{h} Q R_{x h}$ & 194.1562 & 185.5206 & 193.6485 & 143.7761 & 193.1517 & 191.9753 & 383.5274 & 415.2221 & 407.5903 & 102.9615 \\
\hline$\sum_{h=1}^{L} W_{h} M R_{x h}$ & $\sum_{h=1}^{L} W_{h} Q D_{x h}$ & 194.1562 & 185.5206 & 193.6484 & 143.7756 & 193.1516 & 191.9753 & 383.5266 & 415.2217 & 407.5889 & 102.9594 \\
\hline
\end{tabular}

TABLE 5: MSEs of $\hat{\bar{Y}}_{s t}, \hat{\bar{Y}}_{C R}$, and $\hat{\bar{Y}}_{C R e g}$ for different sample sizes.

\begin{tabular}{lccc}
\hline & $\operatorname{MSE}\left(\hat{\bar{Y}}_{s t}\right)$ & $\operatorname{MSE}\left(\hat{\bar{Y}}_{C R}\right)$ & MSE $\left(\hat{\bar{Y}}_{C R e g}\right)$ \\
\hline$n=180$ & 2234.0170 & 208.6489 & 191.4028 \\
$n=250$ & 1600.3790 & 155.6986 & 136.6418 \\
$n=350$ & 971.9409 & 95.6147 & 84.1167 \\
\hline
\end{tabular}

TABLE 6: Minimum MSEs of different estimators based on the simulation study $(n=180)$.

\begin{tabular}{|c|c|c|c|c|c|c|c|c|c|c|}
\hline$a_{s t}$ & $b_{s t}$ & $\widehat{\bar{Y}}_{K}$ & $\hat{\bar{Y}}_{K K}$ & $\widehat{\bar{Y}}_{S G}$ & $\widehat{\bar{Y}}_{H S 1}$ & $\widehat{\bar{Y}}_{H S 2}$ & $\hat{\bar{Y}}_{S S 1 R}$ & $\hat{\bar{Y}}_{S S 1 P}$ & $\widehat{\bar{Y}}_{S S 1 R P}$ & $\widehat{\bar{Y}}_{P}$ \\
\hline 1 & 0 & 208.7024 & 182.4218 & 824243 & 171.9060 & 104.0812 & 181.7569 & 163.3593 & 181.1334 & 91.1038 \\
\hline 1 & $\sum_{h=1}^{L} W_{h} \rho_{h}$ & 208.9233 & 182.8256 & 828281 & 172.3738 & 105.0166 & 182.1669 & 163.8692 & 181.5503 & 92.1283 \\
\hline 1 & $\sum_{h=1}^{L} W_{h} C_{x h}$ & 208.1726 & 182.0508 & 182.0533 & 171.5916 & 104.1605 & 181.3923 & 163.0362 & 180.7751 & 91.2563 \\
\hline$\sum_{h=1}^{L} W_{h} C_{x h}$ & $\sum_{h=1}^{L} W_{h} \beta_{2 x h}$ & 206.1250 & 180.3394 & 180.3419 & 169.9015 & 102.4944 & 179.6982 & 161.1364 & 179.0981 & 89.5657 \\
\hline$\sum_{h=1}^{L} W_{h} \beta_{1 x h}$ & $\sum_{h=1}^{L} W_{h} C_{x h}$ & 207.2181 & 181.6087 & 181.6112 & 171.2167 & 104.2205 & 180.9596 & 162.6734 & 180.3525 & 91.4022 \\
\hline$\sum_{h=1}^{L} W_{h} \beta_{1 x h}$ & $\sum_{h=1}^{L} W_{h} \rho_{h}$ & 208.0968 & 181.9132 & 181.9157 & 171.4806 & 104.2386 & 181.2527 & 163.0222 & 180.6335 & 91.3737 \\
\hline 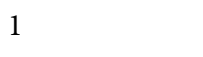 & $\sum_{h=1}^{L} W_{h} \beta_{2 x h}$ & 207.6863 & 181.5076 & 181.5100 & 171.1143 & 104.1185 & 180.8584 & 162.5738 & 180.2520 & 91.2628 \\
\hline 1 & $\sum_{h=1}^{L} W_{h} \beta_{1 x h}$ & 208.9447 & 182.9924 & 182.9949 & 172.5532 & 105.3055 & 182.3371 & 164.0466 & 181.7253 & 92.4328 \\
\hline$\sum_{h=1}^{L} W_{h} T M_{x h}$ & 1 & 208.6489 & 182.8849 & 182.8874 & 172.3985 & 104.8140 & 182.2277 & 163.8243 & 181.6142 & 91.8843 \\
\hline$\sum_{h=1}^{L} W_{h} T M_{x h}$ & $\sum_{h=1}^{L} W_{h} Q R_{x h}$ & 208.9039 & 182.8772 & 182.8797 & 172.4226 & 105.0771 & 182.2188 & 163.9184 & 181.6032 & 92.1900 \\
\hline$\sum_{h=1}^{L} W_{h} Q A_{x h}$ & $\sum_{h=1}^{L} W_{h} S_{x h}$ & 209.1184 & 182.5700 & 182.5725 & 172.0577 & 104.2553 & 181.9039 & 163.516 & 181.2788 & 91.2757 \\
\hline$\sum_{h=1}^{L} W_{h} H L_{x h}$ & $\sum_{h=1}^{L} W_{h} \rho_{h}$ & 208.7805 & 182.6141 & 182.6166 & 172.1151 & 104.4292 & 181.9516 & 163.5377 & 181.3314 & 91.4787 \\
\hline$\sum_{h=1}^{L} W_{h} H L_{x h}$ & $\sum_{h=1}^{L} W_{h} Q R_{x h}$ & 207.4471 & 181.8892 & 181.8914 & 173.1457 & 117.5424 & 181.6167 & 162.7754 & 181.3831 & 105.7468 \\
\hline & & 208.2513 & 181.6575 & 181.66 & 171.1876 & 103.6541 & 180.9891 & 162.7212 & 180.3609 & 90.7320 \\
\hline$\sum_{h=1}^{L} W_{h} M R_{x h}$ & $\sum_{h=1}^{L} W_{h} Q D_{x h}$ & 207.2467 & 181.1462 & 181.1487 & 170.6938 & 103.2264 & 180.4891 & 162.1121 & 179.8724 & 90.3161 \\
\hline
\end{tabular}

Bold values indicate minimum MSE.

TABLE 7: Minimum MSEs of different estimators based on the simulation study $(n=180)$.

\begin{tabular}{lccccccccccc}
\hline$a_{s t}$ & $b_{s t}$ & $\hat{\bar{Y}}_{S S 2 R}$ & $\hat{\bar{Y}}_{S S 2 P}$ & $\hat{\bar{Y}}_{S S 2 R R}$ & $\hat{\bar{Y}}_{S S 2 P P}$ & $\hat{\bar{Y}}_{S S 2 R P}$ & $\hat{\bar{Y}}_{S S 2 P R}$ & $\hat{\bar{Y}}_{S S 31}$ & $\hat{\bar{Y}}_{S S 32}$ & $\hat{\bar{Y}}_{S S 33}$ & $\hat{\bar{Y}}_{P}$ \\
\hline 1 & 0 & 182.3466 & 173.2652 & 182.0005 & 130.4476 & 181.4683 & 179.9313 & 94.73656 & 130.4476 & 160.545 & $\mathbf{9 1 . 1 0 3 8}$ \\
1 & $\sum_{h=1}^{L} W_{h} \rho_{h}$ & 182.7497 & 173.7173 & 182.4081 & 131.1713 & 181.8815 & 180.3422 & 95.68471 & 131.166 & 161.1143 & $\mathbf{9 2 . 1 2 8 3}$ \\
1 & $\sum_{h=1}^{L} W_{h} C_{x h}$ & 181.9698 & 172.9086 & 181.6336 & 130.2683 & 181.1066 & 179.5505 & 94.70196 & 130.261 & 160.3244 & $\mathbf{9 1 . 2 5 6 3}$ \\
$\sum_{h=1}^{L} W_{h} C_{x h}$ & $\sum_{h=1}^{L} W_{h} \beta_{2 x h}$ & 180.2450 & 171.0819 & 179.9328 & 128.2003 & 179.4204 & 177.7797 & 92.33457 & 128.1317 & 158.6125 & $\mathbf{8 9 . 5 6 5 7}$ \\
$\sum_{h=1}^{L} W_{h} \beta_{1 x h}$ & $\sum_{h=1}^{L} W_{h} C_{x h}$ & 181.5270 & 172.4999 & 181.1971 & 130.0811 & 180.6785 & 179.112 & 94.72428 & 130.0791 & 160.0748 & $\mathbf{9 1 . 4 0 2 2}$ \\
$\sum_{h=1}^{L} W_{h} \beta_{1 x h}$ & $\sum_{h=1}^{L} W_{h} \rho_{h}$ & 181.8389 & 172.8398 & 181.4947 & 130.4089 & 180.9661 & 179.4442 & 95.01959 & 130.4074 & 160.221 & $\mathbf{9 1 . 3 7 3 7}$ \\
1 & $\sum_{h=1}^{L} W_{h} \beta_{2 x h}$ & 181.4267 & 172.4007 & 181.0958 & 129.978 & 180.5776 & 179.0127 & 94.42132 & 129.8881 & 159.7761 & $\mathbf{9 1 . 2 6 2 8}$ \\
1 & $\sum_{h=1}^{L} W_{h} \beta_{1 x h}$ & 182.9171 & 173.8882 & 182.5766 & 131.3816 & 182.0538 & 180.5082 & 95.89918 & 131.3617 & 161.3055 & $\mathbf{9 2 . 4 3 2 8}$ \\
\hline
\end{tabular}


TABLE 7: Continued.

\begin{tabular}{|c|c|c|c|c|c|c|c|c|c|c|c|}
\hline$a_{s t}$ & $b_{s t}$ & $\overline{\bar{Y}}_{S S 2 R}$ & $\overline{\bar{Y}}_{S S 2 P}$ & $\hat{\bar{Y}}_{S S 2 R R}$ & $\hat{\bar{Y}}_{S S 2 P P}$ & $\overline{\bar{Y}}_{\text {SS2RP }}$ & $\hat{\bar{Y}}_{S S 2 P R}$ & $\overline{\bar{Y}}_{S S 31}$ & $\overline{\bar{Y}}_{S S 32}$ & $\hat{\bar{Y}}_{S S 33}$ & $\hat{\bar{Y}}_{P}$ \\
\hline$\sum_{h=1}^{L} W_{h} T M_{x h}$ & 1 & 182.8072 & 173.7227 & 182.4678 & 130.9729 & 181.9436 & 180.3827 & 95.33219 & 130.9729 & 161.1424 & 91.8843 \\
\hline$\sum_{h=1}^{L} W_{h} T M_{x h}$ & $\sum_{h=1}^{L} W_{h} Q R_{x h}$ & 182.8017 & 173.7682 & 182.4597 & 131.2176 & 181.9338 & 180.3930 & 95.71564 & 131.208 & 161.1659 & 92.1900 \\
\hline$\sum_{h=1}^{L} W_{h} Q A_{x h}$ & $\sum_{h=1}^{L} W_{h} S_{x h}$ & 182.4934 & 173.4168 & 182.1482 & 130.6215 & 181.6146 & 180.0789 & 94.8953 & 130.6059 & 160.6594 & 91.2757 \\
\hline$\sum_{h=1}^{L} W_{h} H L_{x h}$ & $\sum_{h=1}^{L} W_{h} \rho_{h}$ & 182.5333 & 173.444 & 182.1942 & 130.6498 & 181.6645 & 180.1084 & 94.9620 & 130.6483 & 160.8088 & 91.4787 \\
\hline$\sum_{h=1}^{L} W_{h} H L_{x h}$ & $\sum_{h=1}^{L} W_{h} Q R_{x h}$ & 181.6013 & 172.2849 & 181.7109 & 132.9205 & 181.5076 & 178.6688 & 94.22969 & 129.8961 & 160.2185 & 105.7468 \\
\hline$\sum_{h=1}^{L} W_{h} M R_{x h}$ & $\sum_{h=1}^{L} W_{h} Q R_{x h}$ & 181.5821 & 172.5648 & 181.2344 & 129.9974 & 180.6984 & 179.1876 & 94.48528 & 129.9965 & 159.8306 & 90.7320 \\
\hline$\sum_{h=1}^{L} W_{h} M R_{x h}$ & $\sum_{h=1}^{L} W_{h} Q D_{x h}$ & 181.0637 & 171.9921 & 180.7301 & 129.3164 & 180.2037 & 178.6427 & 93.73929 & 129.316 & 159.4238 & 90.3161 \\
\hline
\end{tabular}
Bold values indicate minimum MSE.

TABLE 8: Minimum MSEs of different estimators based on the simulation study $(n=250)$.

\begin{tabular}{|c|c|c|c|c|c|c|c|c|c|c|}
\hline$a_{s t}$ & $b_{s t}$ & $\hat{\bar{Y}}_{K}$ & $\hat{\bar{Y}}_{K K}$ & $\hat{\bar{Y}}_{S G}$ & $\widehat{\bar{Y}}_{H S 1}$ & $\hat{\bar{Y}}_{H S 2}$ & $\hat{\bar{Y}}_{S S 1 R}$ & $\widehat{\bar{Y}}_{S S 1 P}$ & $\hat{\bar{Y}}_{S S 1 R P}$ & $\hat{\bar{Y}}_{P}$ \\
\hline 1 & 0 & 155.6986 & 130.9425 & 130.9435 & 125.1831 & 87.54561 & 130.5094 & 121.2922 & 130.0932 & 80.3467 \\
\hline 1 & $\sum_{h=1}^{L} W_{h} \rho_{h}$ & 155.6335 & 130.9868 & 130.9878 & 125.2404 & 87.70134 & 130.5545 & 121.3769 & 130.14 & 80.5204 \\
\hline 1 & $\sum_{h=1}^{L} W_{h} C_{x h}$ & 155.188 & 130.6177 & 130.6186 & 124.8729 & 87.3303 & 130.1873 & 120.9742 & 129.7744 & 80.1478 \\
\hline$\sum_{h=1}^{L} W_{h} C_{x h}$ & $\sum_{h=1}^{L} W_{h} \beta_{2 x h}$ & 155.2285 & 130.7593 & 130.7602 & 125.0268 & 87.56625 & 130.3357 & 121.0766 & 129.9308 & 80.3851 \\
\hline$\sum_{h=1}^{L} W_{h} \beta_{1 x h}$ & $\sum_{h=1}^{L} W_{h} C_{x h}$ & 155.3389 & 130.8714 & 130.8724 & 125.1137 & 87.48645 & 130.4407 & 121.2061 & 130.0281 & 80.2890 \\
\hline$\sum_{h=1}^{L} W_{h} \beta_{1 x h}$ & $\sum_{h=1}^{L} W_{h} \rho_{h}$ & 155.6699 & 131.0058 & 131.0068 & 125.244 & 87.60117 & 130.572 & 121.3724 & 130.1561 & 80.4011 \\
\hline & $\sum_{h=1}^{L} W_{h} \beta_{2 x h}$ & 155.0061 & 130.2404 & 130.2413 & 124.5235 & 87.1653 & 129.8145 & 120.6219 & 129.4065 & 79.9985 \\
\hline 1 & $\sum_{h=1}^{L} W_{h} \beta_{1 x h}$ & 155.2016 & 130.5104 & 130.5113 & 124.7728 & 87.28445 & 130.0795 & 120.8933 & 129.6659 & 80.1095 \\
\hline & 1 & 155.5360 & 131.0069 & 131.0078 & 125.2573 & 87.69095 & 130.5764 & 121.3634 & 130.1638 & 80.5060 \\
\hline$\sum_{h=1}^{L} W_{h} T M_{x h}$ & $\sum_{h=1}^{L} W_{h} Q R_{x h}$ & 155.8067 & 131.277 & 131.278 & 125.5701 & 88.33712 & 130.8469 & 121.7497 & 130.4349 & 81.2152 \\
\hline & $\sum_{h=1}^{L} W_{h} S_{x h}$ & 154.6152 & 130.1677 & 130.1686 & 124.4597 & 87.16355 & 129.7406 & 120.5834 & 129.3311 & 80.0265 \\
\hline$\sum_{h=1}^{L} W_{h} H L_{x h}$ & $\sum_{h=1}^{L} W_{h} \rho_{h}$ & 155.7181 & 130.6542 & 130.6551 & 124.9063 & 87.34791 & 130.2172 & 121.0742 & 129.7966 & 80.1639 \\
\hline$\sum_{h=1}^{L} W_{h} H L_{x h}$ & $\sum_{h=1}^{L} W_{h} Q R_{x h}$ & 155.0446 & 130.6161 & 130.6169 & 125.8356 & 95.03376 & 130.4127 & 120.9906 & 130.2424 & 88.5004 \\
\hline$\sum_{h=1}^{L} W_{h} M R_{x h}$ & $\sum_{h=1}^{L} W_{h} \mathrm{Q} R_{x h}$ & 155.8021 & 131.0947 & 131.0957 & 125.365 & 87.96272 & 130.6616 & 121.5326 & 130.2458 & 80.8098 \\
\hline$\sum_{h=1}^{L} W_{h} M R_{x h}$ & $\sum_{h=1}^{L} W_{h} Q D_{x h}$ & 154.8427 & 130.0278 & 130.0287 & 124.2956 & 86.82202 & 129.5947 & 120.4377 & 129.178 & 79.6540 \\
\hline
\end{tabular}

Bold values indicate minimum MSE.

TABLE 9: Minimum MSEs of different estimators based on the simulation study $(n=250)$.

\begin{tabular}{|c|c|c|c|c|c|c|c|c|c|c|c|}
\hline$a_{s t}$ & $b_{s t}$ & $\overline{\bar{Y}}_{S S 2 R}$ & $\hat{\bar{Y}}_{S S 2 P}$ & $\hat{\bar{Y}}_{S S 2 R R}$ & $\hat{\bar{Y}}_{S S 2 P P}$ & $\hat{\bar{Y}}_{S S 2 R P}$ & $\hat{\bar{Y}}_{S S 2 P R}$ & $\overline{\bar{Y}}_{S S 31}$ & $\hat{\bar{Y}}_{S S 32}$ & $\hat{\bar{Y}}_{S S 33}$ & $\hat{\bar{Y}}_{P}$ \\
\hline & 0 & 30.9376 & 126.3729 & 130.671 & 104.1672 & 130.317 & 129.8108 & 85.72231 & 104.1672 & 118.2598 & 80.3467 \\
\hline 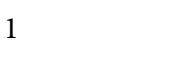 & $\sum_{h=1}^{L} W_{h} \rho_{h}$ & 30.9843 & 26.4391 & 130.7156 & 104.3089 & 130.3628 & 129.8644 & 85.91894 & 104.3061 & 118.3264 & 80.5204 \\
\hline & $\sum_{h=1}^{L} W_{h} C_{x h}$ & 130.612 & 126.0493 & 130.3477 & 103.8761 & 129.9964 & 129.4838 & 85.45359 & 103.8723 & 117.9719 & 80.1478 \\
\hline & $\sum_{h=1}^{L} W_{h} \beta_{2 x h}$ & 130.751 & 126.1653 & 130.4933 & 103.9616 & 130.1484 & 129.61 & 85.45669 & 103.9258 & 118.1125 & 80.3851 \\
\hline & $\sum_{h=1}^{L} W_{h} C_{x h}$ & 130.8666 & 126.2933 & 130.6011 & 104.0666 & 130.2499 & 129.7363 & 85.6053 & 104.0656 & 118.207 & 80.2890 \\
\hline$\sum_{h=1}^{L} W_{h} \beta_{1 x h}$ & $\sum_{h=1}^{L} W_{h} \rho_{h}$ & 131.0036 & 126.4477 & 130.7337 & 104.2572 & 130.3797 & 129.8818 & 85.81913 & 104.2564 & 118.3116 & 80.4011 \\
\hline & $\sum_{h=1}^{L} W_{h} \beta_{2 x h}$ & 130.2341 & 125.6814 & 129.9731 & 103.5868 & 129.6259 & 129.1058 & 85.13934 & 103.5396 & 117.5702 & 79.9985 \\
\hline & $\sum_{h=1}^{L} W_{h} \beta_{1 x h}$ & 130.5056 & 125.9563 & 130.2402 & 103.8306 & 129.8883 & 129.382 & 85.4301 & 103.8202 & 117.8577 & 80.1095 \\
\hline$\sum_{h=1}^{L} W_{h} T M_{x h}$ & 1 & 131.0025 & 126.4398 & 130.7368 & 104.2592 & 130.3856 & 129.8752 & 85.83846 & 104.2592 & 118.3623 & 80.5060 \\
\hline$T M_{x h}$ & $\sum_{h=1}^{L} W_{h} Q R_{x h}$ & 131.2748 & 126.7694 & 131.0072 & 104.829 & 130.6564 & 130.1634 & 86.59144 & 104.824 & 118.7145 & 81.2152 \\
\hline${ }_{h=1}^{L} W_{h} Q A_{x h}$ & $\sum_{h=1}^{L} W_{h} S_{x h}$ & 130.162 & 125.6269 & 129.8997 & 103.5951 & 129.5513 & 129.0399 & 85.28246 & 103.587 & 117.5998 & 80.0265 \\
\hline$\sum_{h=1}^{L} W_{h} H L_{x h}$ & $\sum_{h=1}^{L} W_{h} \rho_{h}$ & 130.6518 & 126.1239 & 130.3805 & 104.0301 & 130.0228 & 129.54 & 85.66646 & 104.0293 & 117.9576 & 80.1639 \\
\hline$\sum_{h=1}^{L} W_{h} H L_{x h}$ & $\sum_{h=1}^{L} W_{h} Q R_{x h}$ & 130.5198 & 125.8475 & 130.4821 & 105.5124 & 130.3329 & 129.135 & 85.57302 & 103.9372 & 118.0266 & 88.5004 \\
\hline
\end{tabular}


TABLE 9: Continued.

\begin{tabular}{|c|c|c|c|c|c|c|c|c|c|c|c|}
\hline$a_{s t}$ & $b_{s t}$ & $\hat{\bar{Y}}_{S S 2 R}$ & $\widehat{\bar{Y}}_{S S 2 P}$ & $\hat{\bar{Y}}_{S S 2 R R}$ & $\hat{\bar{Y}}_{S S 2 P P}$ & $\hat{\bar{Y}}_{S S 2 R P}$ & $\hat{\bar{Y}}_{S S 2 P R}$ & $\hat{\bar{Y}}_{S S 31}$ & $\hat{\bar{Y}}_{S S 32}$ & $\hat{\bar{Y}}_{S S 33}$ & $\overline{\bar{Y}}_{P}$ \\
\hline$\sum_{h=1}^{L} W_{h} M R_{x h}$ & $\sum_{h=1}^{L} W_{h} Q R_{x h}$ & 131.0921 & 126.571 & 130.8231 & 104.5418 & 130.4693 & 129.9783 & 86.23798 & 104.5414 & 118.4748 & 80.8098 \\
\hline$\sum_{h=1}^{L} W_{h} M R_{x h}$ & $\sum_{h=1}^{L} W_{h} Q D_{x h}$ & 130.0233 & 125.4884 & 129.7565 & 103.4035 & 129.4021 & 128.9063 & 85.05503 & 103.4033 & 117.3831 & 79.6540 \\
\hline
\end{tabular}

TABLE 10: Minimum MSEs of different estimators based on the simulation study $(n=350)$.

\begin{tabular}{lcccccccccc}
\hline$a_{s t}$ & $b_{s t}$ & $\hat{\bar{Y}}_{K}$ & $\hat{\bar{Y}}_{K K}$ & $\hat{\bar{Y}}_{S G}$ & $\hat{\bar{Y}}_{H S 1}$ & $\hat{\bar{Y}}_{H S 2}$ & $\hat{\bar{Y}}_{\text {SS1R }}$ & $\hat{\bar{Y}}_{S S 1 P}$ & $\hat{\bar{Y}}_{S S 1 R P}$ & $\hat{\bar{Y}}_{P}$ \\
\hline 1 & 0 & 95.6147 & 80.9165 & 80.9167 & 78.7783 & 64.7223 & 80.7549 & 77.4339 & 80.6023 & $\mathbf{6 2 . 0 3 8 2}$ \\
1 & $\sum_{h=1}^{L} W_{h} \rho_{h}$ & 95.7970 & 81.1781 & 81.1783 & 79.0419 & 65.0101 & 81.0168 & 77.7030 & 80.8649 & $\mathbf{6 2 . 3 3 0 4}$ \\
1 & $\sum_{h=1}^{L} W_{h} C_{x h}$ & 95.8381 & 81.2087 & 81.2089 & 79.0722 & 65.0369 & 81.0475 & 77.7319 & 80.8957 & $\mathbf{6 2 . 3 5 6 4}$ \\
$\sum_{h=1}^{L} W_{h} C_{x h}$ & $\sum_{h=1}^{L} W_{h} \beta_{2 x h}$ & 95.9324 & 81.3256 & 81.3258 & 79.1991 & 65.2374 & 81.1666 & 77.8540 & 81.0173 & $\mathbf{6 2 . 5 6 5 8}$ \\
$\sum_{h=1}^{L} W_{h} \beta_{1 x h}$ & $\sum_{h=1}^{L} W_{h} C_{x h}$ & 95.4390 & 80.8880 & 80.8883 & 78.7582 & 64.7632 & 80.7277 & 77.4170 & 80.5766 & $\mathbf{6 2 . 0 9 0 7}$ \\
$\sum_{h=1}^{L} W_{h} \beta_{1 x h}$ & $\sum_{h=1}^{L} W_{h} \rho_{h}$ & 95.5746 & 80.9982 & 80.9984 & 78.8672 & 64.8656 & 80.8375 & 77.5279 & 80.6861 & $\mathbf{6 2 . 1 9 2 0}$ \\
1 & $\sum_{h=1}^{L} W_{h} \beta_{2 x h}$ & 95.4697 & 80.9304 & 80.9306 & 78.8167 & 64.9358 & 80.7731 & 77.4707 & 80.6256 & $\mathbf{6 2 . 2 7 7 6}$ \\
1 & $\sum_{h=1}^{L} W_{h} \beta_{1 x h}$ & 95.2259 & 80.7899 & 80.7901 & 78.6602 & 64.6624 & 80.6310 & 77.3026 & 80.4815 & $\mathbf{6 1 . 9 8 7 7}$ \\
$\sum_{h=1}^{L} W_{h} T M_{x h}$ & 1 & 95.4491 & 80.9784 & 80.9786 & 78.8435 & 64.8153 & 80.8183 & 77.4931 & 80.6676 & $\mathbf{6 2 . 1 3 6 7}$ \\
$\sum_{h=1}^{L} W_{h} T M_{x h}$ & $\sum_{h=1}^{L} W_{h} Q R_{x h}$ & 95.4708 & 80.9427 & 80.9429 & 78.8079 & 64.7784 & 80.7825 & 77.4580 & 80.6317 & $\mathbf{6 2 . 0 9 8 6}$ \\
$\sum_{h=1}^{L} W_{h} Q A_{x h}$ & $\sum_{h=1}^{L} W_{h} S_{x h}$ & 95.8493 & 81.2139 & 81.2141 & 79.0756 & 65.0261 & 81.0530 & 77.7292 & 80.9015 & $\mathbf{6 2 . 3 4 2 1}$ \\
$\sum_{h=1}^{L} W_{h} H L_{x h}$ & $\sum_{h=1}^{L} W_{h} \rho_{h}$ & 95.2618 & 80.6549 & 80.6551 & 78.5217 & 64.4964 & 80.4942 & 77.1748 & 80.3426 & $\mathbf{6 1 . 8 1 7 9}$ \\
$\sum_{h=1}^{L} W_{h} H L_{x h}$ & $\sum_{h=1}^{L} W_{h} Q R_{x h}$ & 95.3351 & 80.8718 & 80.8720 & 79.0984 & 67.6261 & 80.7971 & 77.4132 & 80.7369 & $\mathbf{6 5 . 1 9 6 8}$ \\
$\sum_{h=1}^{L} W_{h} M R_{x h}$ & $\sum_{h=1}^{L} W_{h} Q R_{x h}$ & 95.3490 & 80.7720 & 80.7722 & 78.6452 & 64.6708 & 80.6114 & 77.3103 & 80.4601 & $\mathbf{6 2 . 0 0 2 3}$ \\
$\sum_{h=1}^{L} W_{h} M R_{x h}$ & $\sum_{h=1}^{L} W_{h} Q D_{x h}$ & 95.4295 & 80.9228 & 80.9230 & 78.7999 & 64.8580 & 80.7628 & 77.4671 & 80.6122 & $\mathbf{6 2 . 1 9 6 0}$ \\
\hline$B$
\end{tabular}

Bold values indicate minimum MSE.

TABLE 11: Minimum MSEs of different estimators based on the simulation study $(n=350)$.

\begin{tabular}{|c|c|c|c|c|c|c|c|c|c|c|c|}
\hline$a_{s t}$ & $b_{s t}$ & $\widehat{\bar{Y}}_{S S 2 R}$ & $\hat{\bar{Y}}_{S S 2 P}$ & $\hat{\bar{Y}}_{S S 2 R R}$ & $\widehat{\bar{Y}}_{S S 2 P P}$ & $\widehat{\bar{Y}}_{S S 2 R P}$ & $\widehat{\bar{Y}}_{S S 2 P R}$ & $\widehat{\bar{Y}}_{S S 31}$ & $\widehat{\bar{Y}}_{S S 32}$ & $\widehat{\bar{Y}}_{S S 33}$ & $\hat{\bar{Y}}_{P}$ \\
\hline 1 & 0 & & & & & & & & & & \\
\hline 1 & $\sum_{h=1}^{L} W_{h} \rho_{h}$ & 1.1856 & 9.5382 & 81.0763 & 1.5339 & - & 80.7866 & 4.9508 & 1.5329 & 76.4450 & 2.3304 \\
\hline 1 & & 81.2162 & 79.5679 & 811069 & 71.5607 & 80.9771 & 808169 & 64.9747 & 715593 & 76.4743 & 623564 \\
\hline${ }_{=1} W_{h} C_{x h}$ & $\sum_{h=1}^{L} W_{h} \beta_{2 x h}$ & & 79.6859 & 81.2251 & 71.7037 & 81.0974 & 80.9320 & 65.1163 & 71.6908 & 76.5992 & 62.5658 \\
\hline${ }_{h=1}^{L} W_{h} \beta_{1 x h}$ & $\sum_{h=1}^{L} W_{h} C_{x h}$ & 0.8951 & 79.2493 & 80.7868 & 1.2598 & 80.6576 & 80.4959 & 64.6915 & 71.2595 & 76.1724 & 62.0907 \\
\hline$\sum_{h=1}^{L} W_{h} \beta_{1 x h}$ & $\sum_{h=1}^{L} W_{h} \rho_{h}$ & 81.0054 & 79.3602 & 80.8967 & 71.3700 & 80.7673 & 80.6066 & 64.8009 & 71.3698 & 76.2789 & 62.1920 \\
\hline 1 & & 80.9370 & 79.2952 & 80.8309 & 71.3489 & 80.7047 & & 64.7841 & 71.3320 & 76.2272 & 62.2776 \\
\hline 1 & & 00.1701 & 79.1 & 80.6895 & 71.1302 & 80.5617 & 80.3 & & 54 & & 61.9877 \\
\hline & 1 & 80.9853 & 79.3323 & 80.8772 & 1.3154 & 80.74 & 80.58 & & 54 & & 62.1367 \\
\hline$\sum_{h=1}^{L} W_{h} T M_{x h}$ & $\sum_{h=1}^{L} W_{h} Q R_{x h}$ & 80.9496 & 79.2969 & 80.8415 & 71.2807 & 80.7126 & 80.5483 & 64.6881 & 71.2788 & 76.2159 & 62.0986 \\
\hline$\sum_{h=1}^{L} W_{h} Q A_{x h}$ & $\sum_{h=1}^{L} W_{h} S_{x h}$ & 81.2212 & 79.5689 & 81.1123 & 71.5478 & 80.9828 & 80.8204 & & 71.5449 & 76.4741 & 62.3421 \\
\hline$\sum_{h=1}^{L} W_{h} H L_{x h}$ & $\sum_{h=1}^{L} W_{h} \rho_{h}$ & 80.6616 & 79.0115 & 80.5535 & 71.0028 & 80.4239 & 80.2613 & 64.4191 & 71.0025 & 75.9286 & 61.8179 \\
\hline$\sum_{h=1}^{L} W_{h} H L_{x h}$ & $\sum_{h=1}^{L} W_{h} Q R_{x h}$ & 80.8480 & 79.1645 & 80.8219 & 71.8426 & 80.7687 & 80.3565 & 64.7315 & 71.2769 & 76.1725 & 65.1968 \\
\hline & & 80.7793 & 79.1383 & 80.6707 & 71.1661 & 80.5413 & 80.3818 & 64.6116 & 71.1659 & 76.0604 & 62.0023 \\
\hline$\sum_{h=1}^{L} W_{h} M R_{x h}$ & ${ }_{h=1}^{L} W_{h} Q D_{x h}$ & 80.9301 & 79.2917 & 80.8218 & 71.3356 & 80.6930 & 80.5328 & 64.7949 & 71.3355 & 76.2251 & 62.1960 \\
\hline
\end{tabular}

Bold values indicate minimum MSE. 
as compared to other estimators. A simulation study is also carried out in support of efficient proposed estimators. So, to get more enhanced results in practice under stratified random sampling, our suggested family of estimators is recommended.

The possible extensions of this work are to estimate the (1) finite population mean using robust quantile regression and L-moments characteristics of an auxiliary information under stratified ranked set sampling, (2) finite population parameters including median, variance, and proportions using L-moments under different sampling designs, and (3) population mean in the presence of nonsampling errors using L-moments and calibration approach.

\section{Data Availability}

The data used to support the findings of this study are included within the article.

\section{Conflicts of Interest}

The authors declare that they have no conflicts of interest.

\section{References}

[1] C. Kadilar and H. Cingi, "Ratio estimator in stratified random sampling," Biometrical Journal, vol. 45, pp. 218-225, 2003.

[2] C. Kadilar and H. Cingi, "A new estimator in stratified random sampling," Communications in Statistics-Theory and Methods, vol. 34, pp. 597-602, 2005.

[3] N. Koyuncu and C. Kadilar, "Ratio and product estimators in stratified random sampling," Journal of Statistical Planning and Inference, vol. 139, pp. 2552-2558, 2009.

[4] N. Koyuncu and C. Kadilar, "On improvement in estimating population mean in stratified random sampling," Journal of Applied Statistics, vol. 37, no. 6, pp. 999-1013, 2010.

[5] H. P. Singh and G. K. Vishwakarma, "Combined ratioproduct estimator of finite population mean in stratified random sampling," Matodo De Encue, vol. 8, pp. 35-44, 2006.

[6] H. P. Singh and G. K. Vishwakarma, "A family of estimators of population mean using auxiliary information in stratified random sampling," Communications in Statistics-Theory and Methods, vol. 37, pp. 1038-1050, 2008.

[7] H. P. Singh and G. K. Vishwakarma, "A general procedure for estimating the population mean in stratified random sampling using auxiliary information," Metron, vol. 62, no. 1, pp. 47-65, 2010.

[8] J. Shabbir and S. Gupta, "On estimating finite population mean in simple and stratified random sampling," Communications in Statistics-Theory and Methods, vol. 40, no. 2, pp. 199-212, 2011.

[9] A. Haq and J. Shabbir, "Improved family of ratio estimators in simple and stratified random sampling," Communications in Statistics - Theory and Methods, vol. 42, no. 5, pp. 782-799, 2013.

[10] R. S. Solanki and H. P. Singh, "An efficient class of estimators for the population mean using auxiliary information in stratified random sampling," Communications in StatisticsTheory and Methods, vol. 43, pp. 3380-3401, 2014.

[11] R. Yadav, L. N. Upadhyaya, H. P. Singh, and S. Chatterjee, "Improved ratio and product exponential type estimators for finite population mean in stratified random sampling,"
Communications in Statistics-Theory and Methods, vol. 43, no. 15, pp. 3269-3285, 2014.

[12] R. S. Solanki and H. P. Singh, "An improved estimation in stratified random sampling," Communications in StatisticsTheory and Methods, vol. 45, no. 7, pp. 2056-2070, 2016.

[13] M. Javed, M. Irfan, and T. Pang, "Hartley- Ross type unbiased estimators of population mean using two auxiliary variables," Scientia Iranica: Transactions on Industrial Engineering (E), vol. 26, no. 6, pp. 3835-3845, 2019.

[14] M. Javed and M. Irfan, “A simulation study: new optimal estimators for population mean by using dual auxiliary information in stratified random sampling," Journal of Taibah University for Science, vol. 14, no. 1, pp. 557-568, 2020.

[15] J. R. Hosking, "L-moments: analysis and estimation of distributions using linear combinations of order statistics," Journal of the Royal Statistical Society: Series B, vol. 52, no. 1, pp. 105-124, 1990.

[16] U. Shahzad, I. Ahmad, I. M. Almanjahie, and N. H. Al-Noor, "L-moments based calibrated variance estimators using double stratified sampling," Computers, Materials \& Continua, vol. 68, no. 3, pp. 3411-3430, 2021.

[17] S. Bahl and R. K. Tuteja, "Ratio and product type exponential estimator," Information and Optimization Sciences, vol. 12, no. 1, pp. 159-163, 1991.

[18] G. Gupta and J. Shabbir, "On improvement in estimating the population mean in simple random sampling," Journal of Applied Statistics, vol. 35, no. 2, pp. 559-566, 2008.

[19] H. P. Singh and R. S. Solanki, "Efficient ratio and product estimators in stratified random sampling," Communications in Statistics-Theory and Methods, vol. 42, pp. 1008-1023, 2013.

[20] J. Shabbir, A. Haq, and S. Gupta, "A new difference-cumexponential type estimator of finite population mean in simple random sampling," Revista Colombiana de Estadística, vol. 37, no. 1, pp. 199-211, 2014.

[21] T. Hettmansperger and J. W. McKean, Robust Nonparametric Statistical Methods, Chapman \& Hall /CRC Press, Boca Raton, FL, USA, 2nd edition, 2011.

[22] T. Wang, Y. Li, and H. Cui, "On weighted randomly trimmed means," Journal of Systems Science and Complexity, vol. 20, no. 1, pp. 47-65, 2007.

[23] M. Irfan, M. Javed, and Z. Lin, "Optimized estimation for population mean using conventional and non-conventional measures under the joint influence of measurement error and non-response," Journal of Statistical Computation and Simulation, vol. 88, no. 12, pp. 2385-2403, 2018.

[24] M. Irfan, M. Javed, and Z. Lin, "Efficient ratio-type estimators of finite population mean based on correlation coefficient," Scientia Iranica: Transactions on Industrial Engineering (E), vol. 25, no. 4, pp. 2361-2372, 2018 b.

[25] M. Irfan, M. Javed, and Z. Lin, "Improved estimation of population mean through known conventional and nonconventional measures of auxiliary variable," Iranian Journal of Science and Technology Transaction A-Science, vol. 43, no. 4, pp. 1851-1862, 2019.

[26] M. Irfan, M. Javed, and S. H. Bhatti, "Difference-type-exponential estimators based on dual auxiliary information under simple random sampling," Scientia Iranica: Transactions on Industrial Engineering (E), 2020, In press. 